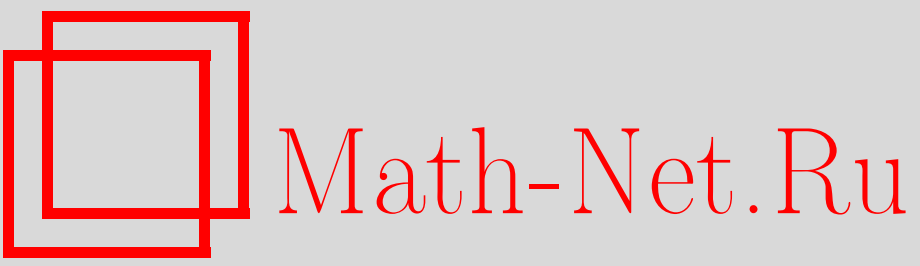

Ю. А. Марков, М. А. Маркова, Калибровочная инвариантность декремента нелинейного затухания Ландау бозе-возбуждений в кварк-глюонной плазме. I, TMФ, 2000, том 124, номер 1, 110-135

DOI: https://doi.org/10.4213/tmf629

Использование Общероссийского математического портала Math-Net.Ru подразумевает, что вы прочитали и согласны с пользовательским соглашением

http://www.mathnet.ru/rus/agreement

Параметры загрузки:

IP: 35.173 .219 .149

26 апреля 2023 г., 02:27:20 


\author{
ТЕОРЕТИЧЕСКАЯ \\ И МАТЕМАТИЧЕСКАЯ \\ ФИЗИКА \\ Том 124, № 1 \\ июль, 2000
}

(C) 2000 г.

Ю. А. Марков* , М.А. Маркова*

\title{
КАЛИБРОВОЧНАЯ ИНВАРИАНТНОСТЬ ДЕКРЕМЕНТА НЕЛИНЕЙНОГО ЗАТУХАНИЯ ЛАНДАУ БОЗЕ-ВОЗБУЖДЕНИЙ В КВАРК-ГЛЮОННОЙ ПЛАЗМЕ. І
}

На основе уравнения Янга-Миллса и динамических уравнений, описывающих поведение кварк-глюонной плазмы в квазиклассическом пределе, получено кинетическое уравнение для продольных волн (плазмонов) с учетом их взаимодействия. С помощью эффективных тождеств Уорда доказана калибровочная инвариантность найденного декремента нелинейного затухания Ландау.

\section{1. ВВЕДЕНИЕ}

В последние два десятилетия наблюдается неослабевающий интерес к теоретическим исследованиям свойств нового фундаментального состояния материи - кварк-глюонной плазмы (КГП), что связано с интенсивными ее поисками в экспериментах по соударению ультрарелятивистских тяжелых ионов.

В качестве основных методов исследования неравновесных явлений в КГП используются метод температурных функций Грина и кинетический подход. Значительный прогресс был достигнут в развитии первого из них. На основе ресуммирования так называемых жестких температурных петель (HTL) была построена эффективная пертурбативная теория $[1,2]$ и разрешен вопрос о знаке и калибровочной зависимости декремента затухания мягких возбуждений КГП. В работе [3] вычислен декремент затухания для тяжелого фермиона, в работах [4-6] - для мягких плазмона и плазмино. Расширение программы ресуммирования путем включения эффектов более высокого порядка в жесткие пропагаторы позволило также вычислить декременты затухания для энергичных фермионов и бозонов $[7,8]$. Прогресс в высокотемпературной (когда пренебрегаем массами кварков по сравнению с температурой) квантовой хромодинамике (КХД) позволил по-новому взглянуть на уже имеющуюся кинетическую теорию КГП, основы которой были заложены в работах [9-14], и дал толчок к ее дальнейшему развитию.

${ }^{*}$ Институт динамики систем и теории управления СО РАН, Иркутск, Россия. E-mail: markov@icc.ru 
Несмотря на то что язык и методы этих двух подходов очень различны, тем не менее сушествует тесная связь между HTL-приближением и транспортной теорией. Первоначально Силин использовал кинетическую теорию для вывода HTL в собственно-энергетической функции фотона [15]. HTL в кварковой и глюонной собственно-энергетических функциях могут быть вычислены подобным образом, и даже, более того, в работе [16] показано, что функционал, порождаюший HTL с произвольным числом внешних мягких бозонных линий, может быть выведен из классической кинетической теории КГП, что явно указывает на классический характер жестких температурных эффектов. Декремент затухания жестких возмушений в КГП вычислен в работе [17] на основе уравнения больцмановского типа со столкновительным членом.

Дальнейший шаг в развитии кинетической теории был сделан в работах $[18,19]$. В отличие от ранних работ по транспортной теории КГП [9-14], здесь с самого начала при выводе кинетических уравнений в полной мере используются представления, развитые в высокотемпературной КХД. Полученные уравнения выделяют согласованно доминируюшие по константе взаимодействия $g$ члены в иерархии уравнений, которые описывают отклик плазмы на слабые и слабоменяюшиеся возмушения и учитывают все эффекты, связанные с HTL. Однако здесь необходимо заметить, что если пренебречь влиянием усредненного фермионного поля, то полученное в работе [18] нелинейное уравнение, описывающее нормальные бозонные моды плазмы, полностью совпадет с соответствующим уравнением, полученным в работе [16] на основе обычной классической кинетической теории. Это в известной степени оправдывает использование классических и квазиклассических кинетических уравнений, найденных в работах [9-14], несмотря на то что промежуточные аппроксимационные схемы, с помошью которых они были получены, смешивают лидируюшие и нелидирующие вклады по константе $g$, которые, таким образом, являются внутренне несогласованными.

Такое тесное переплетение двух методов исследования неравновесных явлений в КГП естественно приводит к вопросу: можно ли, оставаясь в рамках только классической (квазиклассической) кинетической теории, вычислить декремент затухания мягких бозонных мод, соответствующий приближению HTL [4]. Впервые такой вопрос был поставлен в работе [20], и на основе полученных результатов был дан положительный ответ.

В статье [21] было показано, что в КГП затухание Ландау в линейном приближении отсутствует. Поэтому, как было справедливо замечено в работе [20], фактически единственным механизмом, с которым можно связать затухание из кинетической теории с затуханием из HTL-приближения, является нелинейное затухание Ландау, связанное с нелинейными эффектами взаимодействия волн и частиц в КГП. Для выяснения этой связи в [20] был использован многовременной метод, развитый в свое время применительно к изучению нелинейных свойств электрон-ионной (абелевой) плазмы [22]. С помощью этого метода в [20] вычислен нелинейный сдвиг собственной частоты продольных колебаний (в $A_{0}$-калибровке), мнимая часть которого и определяет искомый декремент нелинейного затухания Ландау. Далее было определено предельное выражение 
найденного декремента для $(\mathbf{k}=0)$-моды и сделан численный расчет для приближенной оценки. Полученное таким образом значение оказалось в согласии с соответствующим численным значением, найденным в работе [4] на основе эффективной пертурбативной теории.

Однако при внимательном разборе работы [20] нами были обнаружены некоторые неточности в вычислении как принципиального, так и непринщипиального характера, устранение которых в конечном итоге приводит не только к численному изменению найденного в работе [20] предельного значения декремента нелинейного затухания Ландау, но и, что самое главное, меняет его знак на противоположный. Это указывает на некоторую преждевременность утверждений о найденной связи декремента нелинейного затухания Ландау с декрементом затухания из HTL-приближения.

Кроме того, в работе [20] не были вскрыты физические механизмы, лежащие в основе процесса нелинейного рассеяния продольных волн на частищах КГП, а вклад в данньй процесс от эффекта самодействия калибровочного поля вовсе был отброшен, хотя в “мягкой” области возбуждений он имеет тот же порядок, что и вклады, связанные с эффектами среды. Не был также рассмотрен принципиально важный вопрос о калибровочной зависимости полученного выражения декремента нелинейного затухания Ландау. В связи с замечаниями, с деланными выше, ясно, что данньй вопрос требует дополнительного тщательного исследования.

В этой работе мы рассматриваем вышеприведенную задачу, используя в качестве основного метода исследования нелинейных процессов в КГП подход, основанный на получении обобшенного кинетического (или просто кинетического) уравнения для волн в КГП, развитый в свое время в работах [23-27] применительно к абелевой плазме. Нами показано, что декремент нелинейного затухания Ландау $\gamma^{l}(\mathbf{k})$ продольных волн в КГП определяет фактически два различных процесса: эффективное перекачивание энергии по спектру от коротких волн к длинным (при полном сохранении энергии возбуждения) и собственно нелинейное поглощение плазменных волн частицами КГП. Основной вывод, сделанный нами в этой работе (он будет опубликован во второй части данной статьи, в разделе 2), состоит в том, что с декрементом затухания мягких бозонных мод из HTL-приближения [4] необходимо сравнивать ту часть $\gamma^{l}(0)$, которая отвечает за нелинейное поглощение волн и является положительной для любого значения волнового вектора $\mathbf{k}$ и, в частности, для $(\mathbf{k}=0)$-моды.

\section{2. ИСХОДНЫЕ УРАВНЕНИЯ. МЕТОД УСРЕДНЕНИЯ ПО СТАТИСТИЧЕСКОМУ АНСАМБЛЮ}

Мы используем метрику $g^{\mu \nu}=\operatorname{diag}(1,-1,-1,-1)$ и выбираем систему единиц такую, что $c=k_{\mathrm{B}}=1$. Потенциалы калибровочного поля, являющиеся $\left(N_{\mathrm{c}} \times N_{\mathrm{c}}\right)$-матрицами в цветовом пространстве, определены посредством соотношения $A_{\mu}=A_{\mu}^{a} t^{a}$ с $N_{\mathrm{c}}^{2}-1$ эрмитовыми генераторами группы $S U\left(N_{\mathrm{c}}\right)$ в фундаментальном представлении. 
Тензор напряженности поля $F_{\mu \nu}=F_{\mu \nu}^{a} t^{a}$, где

$$
F_{\mu \nu}^{a}=\partial_{\mu} A_{\nu}^{a}-\partial_{\nu} A_{\mu}^{a}+g f^{a b c} A_{\mu}^{b} A_{\nu}^{c},
$$

подчиняется уравнению Янга-Миллса в ковариантной калибровке

$$
\partial_{\mu} F^{\mu \nu}(X)-i g\left[A_{\mu}(X), F^{\mu \nu}(X)\right]-\xi^{-1} \partial^{\nu} \partial^{\mu} A_{\mu}(X)=-j^{\nu}(X),
$$

где $\xi$ - калибровочный параметр, $j^{\nu}$ - цветной ток, определяемый как

$$
j^{\nu}=g t^{a} \int d^{4} p p^{\nu}\left[\operatorname{Sp} t^{a}\left(f_{\mathrm{q}}-f_{\overline{\mathrm{q}}}\right)+\operatorname{Tr}\left(T^{a} f_{\mathrm{g}}\right)\right]
$$

Здесь $T^{a}$ - эрмитовы генераторы $S U\left(N_{\mathrm{c}}\right)$-группы в присоединенном представлении $\left(\left(T^{a}\right)^{b c}=-i f^{a b c}, \operatorname{Tr}\left(T^{a} T^{b}\right)=N_{\mathrm{c}} \delta^{a b}\right)$. Значок $\operatorname{Tr}$ обозначает след по цветным индексам в присоединенном представлении. Функции распределения кварков $f_{\mathrm{q}}$ (антикварков $\left.f_{\overline{\mathrm{q}}}\right)$ и глюонов $f_{\mathrm{g}}$ являются соответственно $\left(N_{\mathrm{c}} \times N_{\mathrm{c}}\right)$ - и $\left(N_{\mathrm{c}}^{2}-1\right) \times\left(N_{\mathrm{c}}^{2}-1\right)$-эрмитовыми матрицами в цветовом пространстве и удовлетворяют динамическим уравнениям, которые в квазиклассическом пределе имеют вид $[12,13]$

$$
\begin{aligned}
p^{\mu} \mathcal{D}_{\mu} f_{\mathrm{q}, \overline{\mathrm{q}}} \pm \frac{1}{2} g p^{\mu}\left\{F_{\mu \nu}, \frac{\partial f_{\mathrm{q}, \overline{\mathrm{q}}}}{\partial p_{\nu}}\right\} & =0, \\
p^{\mu} \widetilde{\mathcal{D}}_{\mu} f_{\mathrm{g}}+\frac{1}{2} g p^{\mu}\left\{\mathcal{F}_{\mu \nu}, \frac{\partial f_{\mathrm{g}}}{\partial p_{\nu}}\right\} & =0,
\end{aligned}
$$

где $\mathcal{D}_{\mu}$ и $\widetilde{\mathcal{D}}_{\mu}$ - ковариантные производные, которые действуют следуюшим образом:

$$
\begin{aligned}
& \mathcal{D}_{\mu}=\partial_{\mu}-i g\left[A_{\mu}(X), \cdot\right], \\
& \widetilde{\mathcal{D}}_{\mu}=\partial_{\mu}-i g\left[\mathcal{A}_{\mu}(X), \cdot\right],
\end{aligned}
$$

$[$,$] обозначает коммутатор, \{$,$\} - антикоммутатор и \mathcal{A}_{\mu}, \mathcal{F}_{\mu \nu}$ определены как $\mathcal{A}_{\mu}=$ $A_{\mu}^{a} T^{a}, \mathcal{F}_{\mu \nu}=F_{\mu \nu}^{a} T^{a}$. Верхний знак в первом уравнении (2.4) относится к кваркам, а нижний - к антикваркам. В данной работе мы пренебрегаем спиновой степенью свободы частиц КГП, которую, однако, несложно учесть в данном приближении на уровне исходных уравнений для функций распределений (2.4) (см., например, [14]).

Наше рассмотрение начнем с динамических, обратимых уравнений (2.4). Разобьем функции распределения $f_{\mathrm{q}, \overline{\mathrm{q}}}$ и $f_{\mathrm{g}}$ на две части - регулярную и случайную (турбулентную):

$$
f_{\mathrm{s}}=f_{\mathrm{s}}^{\mathrm{R}}+f_{\mathrm{s}}^{\mathrm{T}}, \quad \mathrm{s}=\mathrm{q}, \overline{\mathrm{q}}, \mathrm{g},
$$

так, что

$$
\left\langle f_{\mathrm{s}}\right\rangle=f_{\mathrm{s}}^{\mathrm{R}}, \quad\left\langle f_{\mathrm{s}}^{\mathrm{T}}\right\rangle=0,
$$


где $\langle\cdot\rangle$ обозначает усреднение по статистическому ансамблю, в качестве которого будем рассматривать начальные значения фаз колебаний.

По определению положим также

$$
A_{\mu}=A_{\mu}^{\mathrm{R}}+A_{\mu}^{\mathrm{T}}, \quad\left\langle A_{\mu}^{\mathrm{T}}\right\rangle=0 .
$$

Далее регулярную часть поля $A_{\mu}^{\mathrm{R}}$ будем считать равной нулю.

Усреднив уравнения (2.4) по статистическому ансамблю, с учетом формул (2.5)-(2.7) получим уравнения на регулярную часть функций распределения $f_{\mathrm{q}, \overline{\mathrm{q}}}^{\mathrm{R}}$ и $f_{\mathrm{g}}^{\mathrm{R}}$ :

$$
\begin{aligned}
p^{\mu} \partial_{\mu} f_{\mathrm{q}, \overline{\mathrm{q}}}^{\mathrm{R}}= & i g p^{\mu}\left\langle\left[A_{\mu}^{\mathrm{T}}, f_{\mathrm{q}, \overline{\mathrm{q}}}^{\mathrm{T}}\right]\right\rangle \mp \frac{1}{2} g p^{\mu}\left\langle\left\{\left(F_{\mu \nu}^{\mathrm{T}}\right)_{\mathrm{L}}, \frac{\partial f_{\mathrm{q}, \overline{\mathrm{q}}}^{\mathrm{T}}}{\partial p_{\nu}}\right\}\right\rangle \mp \\
& \mp \frac{1}{2} g p^{\mu}\left\{\left\langle\left(F_{\mu \nu}^{\mathrm{T}}\right)_{\mathrm{NL}}\right\rangle, \frac{\partial f_{\mathrm{q}, \overline{\mathrm{q}}}^{\mathrm{R}}}{\partial p_{\nu}}\right\} \mp \frac{1}{2} g p^{\mu}\left\langle\left\{\left(F_{\mu \nu}^{\mathrm{T}}\right)_{\mathrm{NL}}, \frac{\partial f_{\mathrm{q}, \overline{\mathrm{q}}}^{\mathrm{T}}}{\partial p_{\nu}}\right\}\right\rangle, \\
p^{\mu} \partial_{\mu} f_{\mathrm{g}}^{\mathrm{R}}= & i g p^{\mu}\left\langle\left[\mathcal{A}_{\mu}^{\mathrm{T}}, f_{\mathrm{g}}^{\mathrm{T}}\right]\right\rangle-\frac{1}{2} g p^{\mu}\left\langle\left\{\left(\mathcal{F}_{\mu \nu}^{\mathrm{T}}\right)_{\mathrm{L}}, \frac{\partial f_{\mathrm{g}}^{\mathrm{T}}}{\partial p_{\nu}}\right\}\right\rangle- \\
& -\frac{1}{2} g p^{\mu}\left\{\left\langle\left(\mathcal{F}_{\mu \nu}^{\mathrm{T}}\right)_{\mathrm{NL}}\right\rangle, \frac{\partial f_{\mathrm{g}}^{\mathrm{R}}}{\partial p_{\nu}}\right\}-\frac{1}{2} g p^{\mu}\left\langle\left\{\left(\mathcal{F}_{\mu \nu}^{\mathrm{T}}\right)_{\mathrm{NL}}, \frac{\partial f_{\mathrm{g}}^{\mathrm{T}}}{\partial p_{\nu}}\right\}\right\rangle .
\end{aligned}
$$

Здесь индексы $\mathrm{L}$ и NL обозначают линейную и нелинейную по полю $A_{\mu}^{a}$ части тензора напряженности (2.1). Вычитая далее $(2.8)$ из $(2.4)$, получаем уравнения на $f_{\mathrm{q}, \overline{\mathrm{q}}}^{\mathrm{T}}$ и $f_{\mathrm{g}}^{\mathrm{T}}$ :

$$
\begin{aligned}
p^{\mu} \partial_{\mu} f_{\mathrm{q}, \overline{\mathrm{q}}}^{\mathrm{T}}= & i g p^{\mu}\left(\left[A_{\mu}^{\mathrm{T}}, f_{\mathrm{q}, \overline{\mathrm{q}}}^{\mathrm{T}}\right]-\left\langle\left[A_{\mu}^{\mathrm{T}}, f_{\mathrm{q}, \overline{\mathrm{q}}}^{\mathrm{T}}\right]\right) \mp \frac{1}{2} g p^{\mu}\left\{\left(F_{\mu \nu}^{\mathrm{T}}\right)_{\mathrm{L}}, \frac{\partial f_{\mathrm{q}, \overline{\mathrm{q}}}^{\mathrm{R}}}{\partial p_{\nu}}\right\} \mp\right. \\
& \frac{1}{2} g p^{\mu}\left(\left\{\left(F_{\mu \nu}^{\mathrm{T}}\right)_{\mathrm{L}}, \frac{\partial f_{\mathrm{q}, \overline{\mathrm{q}}}^{\mathrm{T}}}{\partial p_{\nu}}\right\}-\left\langle\left\{\left(F_{\mu \nu}^{\mathrm{T}}\right)_{\mathrm{L}}, \frac{\partial f_{\mathrm{q}, \overline{\mathrm{q}}}^{\mathrm{T}}}{\partial p_{\nu}}\right\}\right\rangle\right) \mp \\
& \mp \frac{1}{2} g p^{\mu}\left\{\left(F_{\mu \nu}^{\mathrm{T}}\right)_{\mathrm{NL}}-\left\langle\left(F_{\mu \nu}^{\mathrm{T}}\right)_{\mathrm{NL}}\right\rangle, \frac{\partial f_{\mathrm{q}, \overline{\mathrm{q}}}^{\mathrm{R}}}{\partial p_{\nu}}\right\} \mp \\
& \mp \frac{1}{2} g p^{\mu}\left(\left\{\left(F_{\mu \nu}^{\mathrm{T}}\right)_{\mathrm{NL}}, \frac{\partial f_{\mathrm{q}, \overline{\mathrm{q}}}^{\mathrm{T}}}{\partial p_{\nu}}\right\}-\left\langle\left\{\left(F_{\mu \nu}^{\mathrm{T}}\right)_{\mathrm{NL}}, \frac{\partial f_{\mathrm{q}, \overline{\mathrm{q}}}^{\mathrm{T}}}{\partial p_{\nu}}\right\}\right\rangle\right), \\
p^{\mu} \partial_{\mu} f_{\mathrm{g}}^{\mathrm{T}}= & i g p^{\mu}\left(\left[\mathcal{A}_{\mu}^{\mathrm{T}}, f_{\mathrm{g}}^{\mathrm{T}}\right]-\left\langle\left[\mathcal{A}_{\mu}^{\mathrm{T}}, f_{\mathrm{g}}^{\mathrm{T}}\right]\right\rangle\right)-\frac{1}{2} g p^{\mu}\left\{\left(\mathcal{F}_{\mu \nu}^{\mathrm{T}}\right)_{\mathrm{L}}, \frac{\partial f_{\mathrm{g}}^{\mathrm{R}}}{\partial p_{\nu}}\right\}- \\
& -\frac{1}{2} g p^{\mu}\left(\left\{\left(\mathcal{F}_{\mu \nu}^{\mathrm{T}}\right)_{\mathrm{L}}, \frac{\partial f_{\mathrm{g}}^{\mathrm{T}}}{\partial p_{\nu}}\right\}-\left\langle\left\{\left(\mathcal{F}_{\mu \nu}^{\mathrm{T}}\right)_{\mathrm{L}}, \frac{\partial f_{\mathrm{g}}^{\mathrm{T}}}{\partial p_{\nu}}\right\}\right\rangle\right)- \\
& -\frac{1}{2} g p^{\mu}\left\{\left(\mathcal{F}_{\mu \nu}^{\mathrm{T}}\right)_{\mathrm{NL}}-\left\langle\left(\mathcal{F}_{\mu \nu}^{\mathrm{T}}\right)_{\mathrm{NL}}\right\rangle, \frac{\partial f_{\mathrm{g}}^{\mathrm{R}}}{\partial p_{\nu}}\right\}- \\
& -\frac{1}{2} g p^{\mu}\left(\left\{\left(\mathcal{F}_{\mu \nu}^{\mathrm{T}}\right)_{\mathrm{NL}}, \frac{\partial f_{\mathrm{g}}^{\mathrm{T}}}{\partial p_{\nu}}\right\}-\left\langle\left\{\left(\mathcal{F}_{\mu \nu}^{\mathrm{T}}\right)_{\mathrm{NL}}, \frac{\partial f_{\mathrm{g}}^{\mathrm{T}}}{\partial p_{\nu}}\right\}\right\rangle\right) .
\end{aligned}
$$


Система уравнений (2.8) и (2.9) удобна для исследования таких неравновесных процессов в КГП, при которых энергия плазменных колебаний мала по сравнению с энергией частиц. В этом случае можно пользоваться разложением в ряд по степеням амплитуды колебаний осциллирующих функций $f_{\mathrm{s}}^{\mathrm{T}}$,

$$
f_{\mathrm{s}}^{\mathrm{T}}=\sum_{n=1}^{\infty} f_{\mathrm{s}}^{\mathrm{T}(n)}, \quad \mathrm{s}=\mathrm{q}, \overline{\mathrm{q}}, \mathrm{g},
$$

где индекс $n$ показывает, что $f_{\mathrm{s}}^{\mathrm{T}(n)}$ пропорциональна $n$-й степени $A_{\mu}^{\mathrm{T}}$. Подставляя разложения $(2.10)$ в (2.9) и собирая члены одного порядка по $A_{\mu}^{\mathrm{T}}$, получим систему уравнений

$$
\begin{aligned}
p^{\mu} \partial_{\mu} f_{\mathrm{q}, \overline{\mathrm{q}}}^{\mathrm{T}(1)}= & \mp \frac{1}{2} g p^{\mu}\left\{\left(F_{\mu \nu}^{\mathrm{T}}\right)_{\mathrm{L}}, \frac{\partial f_{\mathrm{q}, \overline{\mathrm{q}}}^{\mathrm{R}}}{\partial p_{\nu}}\right\}, \\
p^{\mu} \partial_{\mu} f_{\mathrm{q}, \overline{\mathrm{q}}}^{\mathrm{T}(2)}= & i g p^{\mu}\left(\left[A_{\mu}^{\mathrm{T}}, f_{\mathrm{q}, \overline{\mathrm{q}}}^{\mathrm{T}(1)}\right]-\left\langle\left[A_{\mu}^{\mathrm{T}}, f_{\mathrm{q}, \overline{\mathrm{q}}}^{\mathrm{T}(1)}\right]\right\rangle\right) \mp \\
& \mp \frac{1}{2} g p^{\mu}\left(\left\{\left(F_{\mu \nu}^{\mathrm{T}}\right)_{\mathrm{L}}, \frac{\partial f_{\mathrm{q}, \overline{\mathrm{q}}}^{\mathrm{T}(1)}}{\partial p_{\nu}}\right\}-\left\langle\left\{\left(F_{\mu \nu}^{\mathrm{T}}\right)_{\mathrm{L}}, \frac{\partial f_{\mathrm{q}, \overline{\mathrm{q}}}^{\mathrm{T}(1)}}{\partial p_{\nu}}\right\}\right\rangle\right) \mp \\
& \mp \frac{1}{2} g p^{\mu}\left\{\left(F_{\mu \nu}^{\mathrm{T}}\right)_{\mathrm{NL}}-\left\langle\left(F_{\mu \nu}^{\mathrm{T}}\right)_{\mathrm{NL}}\right\rangle, \frac{\partial f_{\mathrm{q}, \overline{\mathrm{q}}}^{\mathrm{R}}}{\partial p_{\nu}}\right\}, \\
p^{\mu} \partial_{\mu} f_{\mathrm{q}, \overline{\mathrm{q}}}^{\mathrm{T}(3)}= & i g p^{\mu}\left(\left[A_{\mu}^{\mathrm{T}}, f_{\mathrm{q}, \overline{\mathrm{q}}}^{\mathrm{T}(2)}\right]-\left\langle\left[A_{\mu}^{\mathrm{T}}, f_{\mathrm{q}, \overline{\mathrm{q}}}^{\mathrm{T}(2)}\right]\right\rangle\right) \mp \\
& \mp \frac{1}{2} g p^{\mu}\left(\left\{\left(F_{\mu \nu}^{\mathrm{T}}\right)_{\mathrm{L}}, \frac{\partial f_{\mathrm{q}, \overline{\mathrm{q}}}^{\mathrm{T}(2)}}{\partial p_{\nu}}\right\}-\left\langle\left\{\left(F_{\mu \nu}^{\mathrm{T}}\right)_{\mathrm{L}}, \frac{\partial f_{\mathrm{q}, \overline{\mathrm{q}}}^{\mathrm{T}(2)}}{\partial p_{\nu}}\right\}\right\rangle\right) \mp \\
& \mp \frac{1}{2} g p^{\mu}\left(\left\{\left(F_{\mu \nu}^{\mathrm{T}}\right)_{\mathrm{NL}}, \frac{\partial f_{\mathrm{q}, \overline{\mathrm{q}}}^{\mathrm{T}(1)}}{\partial p_{\nu}}\right\}-\left\langle\left\{\left(F_{\mu \nu}^{\mathrm{T}}\right)_{\mathrm{NL}}, \frac{\partial f_{\mathrm{q}, \overline{\mathrm{q}}}^{\mathrm{T}(1)}}{\partial p_{\nu}}\right\}\right\rangle\right),
\end{aligned}
$$

Аналогичные уравнения определяем и для $f_{\mathrm{g}}^{\mathrm{T}(n)}, n=1,2,3, \ldots$.

Отсюда нетрудно найти нелинейный цветной ток, который мы также представим в виде разложения

$$
j_{\mu}=j_{\mu}^{\mathrm{R}}+j_{\mu}^{\mathrm{T}}, \quad\left\langle j_{\mu}\right\rangle=j_{\mu}^{\mathrm{R}}, \quad j_{\mu}^{\mathrm{T}}=\sum_{n=1}^{\infty} j_{\mu}^{\mathrm{T}(n)}
$$

где в силу определения (2.3) и разложений (2.10) имеем

$$
j_{\mu}^{\mathrm{T}(n)}=g t^{a} \int d^{4} p p_{\mu}\left[\mathrm{Sp} t^{a}\left(f_{\mathrm{q}}^{\mathrm{T}(n)}-f_{\overline{\mathrm{q}}}^{\mathrm{T}(n)}\right)+\operatorname{Tr}\left(T^{a} f_{\mathrm{g}}^{\mathrm{T}(n)}\right)\right] .
$$


Обратимся теперь к уравнению Янга-Миллса (2.2), связывающему калибровочное поле с цветным током. Усредняя уравнение (2.2) и вычитая усредненное уравнение из $(2.2)$, с учетом $(2.7)$ и (2.14) находим

$$
\begin{aligned}
& \partial_{\mu}\left(F^{\mathrm{T} \mu \nu}\right)_{\mathrm{L}}-\xi^{-1} \partial^{\nu} \partial^{\mu} A_{\mu}^{\mathrm{T}}+j^{\mathrm{T}(1) \nu}=-j_{\mathrm{NL}}^{\mathrm{T} \nu}+i g \partial_{\mu}\left(\left[A^{\mathrm{T} \mu}, A^{\mathrm{T} \nu}\right]-\left\langle\left[A^{\mathrm{T} \mu}, A^{\mathrm{T} \nu}\right]\right\rangle\right)+ \\
& \quad+i g\left(\left[A_{\mu}^{\mathrm{T}},\left(F^{\mathrm{T} \mu \nu}\right)_{\mathrm{L}}\right]-\left\langle\left[A_{\mu}^{\mathrm{T}},\left(F^{\mathrm{T} \mu \nu}\right)_{\mathrm{L}}\right]\right\rangle\right)+ \\
& \quad+g^{2}\left(\left[A_{\mu}^{\mathrm{T}},\left[A^{\mathrm{T} \mu}, A^{\mathrm{T} \nu}\right]\right]-\left\langle\left[A_{\mu}^{\mathrm{T}},\left[A^{\mathrm{T} \mu}, A^{\mathrm{T} \nu}\right]\right]\right\rangle\right)
\end{aligned}
$$

Здесь в левой части собраны все линейные по $A_{\mu}^{\mathrm{T}}$ члены и обозначены как $j_{\mathrm{NL}}^{\mathrm{T}} \nu$ $j^{\mathrm{T}(2) \nu}+j^{\mathrm{T}(3) \nu}+\cdots$.

Чтобы учесть нелинейное взаимодействие волн и частиц в КГП (в первом неисчезающем приближении по энергии волн), ограничимся в разложении (2.10) слагаемыми, кубичными по амплитуде колебаний. Сделаем еше одно упрошаюшее предположение. Уравнения (2.8) представляют собой обычные кинетические уравнения на усредненные функции распределения. Корреляционные функции в правых частях этих уравнений имеют смысл интегралов столкновений частиц КГП с волнами и описывают влияние плазменных волн на основное состояние.

Предположим, однако, что характерное время нелинейной релаксации колебаний мало по сравнению с временем релаксации распределений частиц $f_{\mathrm{S}}^{\mathrm{R}}$, которые можно считать такими же, как и в отсутствие колебаний. Другими словами, мы пренебрегаем изменением в пространстве и во времени регулярной части функций распределения, считая такие функции заданными и описывающими глобальное равновесие КГП,

$$
f_{\mathrm{q}, \overline{\mathrm{q}}}^{\mathrm{R}} \equiv f_{\mathrm{q}, \overline{\mathrm{q}}}^{0}=2 \frac{2 N_{f} \theta\left(p_{0}\right)}{(2 \pi)^{3}} \delta\left(p^{2}\right) \frac{1}{e^{\frac{p u}{T} \mp \mu}+1}, \quad f_{\mathrm{g}}^{\mathrm{R}} \equiv f_{\mathrm{g}}^{0}=2 \frac{2 \theta\left(p_{0}\right)}{(2 \pi)^{3}} \delta\left(p^{2}\right) \frac{1}{e^{\frac{p u}{T}}-1},
$$

где $N_{f}$ - число типов безмассовых кварков, $u_{\mu}-4$-скорость плазмы, находящейся при температуре $T$, и $\mu$ - кварковый химический потенциал.

\section{3. ЛИНЕЙНОЕ ПРИБЛИЖЕНИЕ. КОРРЕЛЯЦИОННАЯ ФУНКЦИЯ СЛУЧАЙНЫХ КОЛЕБАНИЙ}

Обратимся теперь к выводу кинетического уравнения для волн. Исходным является уравнение (2.16). Левая его часть содержит линейное приближение цветного тока, явный вид которого легко найти из уравнения (2.11). Далее будем использовать импульсное представление. Соответствующие уравнения получаются с помощью равенства

$$
A_{\mu}(X)=\int d^{4} k A_{\mu}(k) e^{-i k X}
$$

и аналогичного преобразования для $f_{\mathrm{q}, \overline{\mathrm{q}}}^{\mathrm{T}}, f_{\mathrm{g}}^{\mathrm{T}}$. Переходя к фурье-компонентам, из $(2.11)$ находим

$$
\begin{gathered}
f_{\mathrm{q}, \overline{\mathrm{q}}}^{\mathrm{T}(1)}(k, p)=\mp g \frac{\chi^{\nu \lambda}(k, p)}{p k+i p_{0} \epsilon} \frac{\partial f_{\mathrm{q}, \overline{\mathrm{q}}}^{0}}{\partial p^{\lambda}} A_{\nu}(k), \quad f_{\mathrm{g}}^{\mathrm{T}(1)}(k, p)=-g \frac{\chi^{\nu \lambda}(k, p)}{p k+i p_{0} \epsilon} \frac{\partial f_{\mathrm{g}}^{0}}{\partial p^{\lambda}} \mathcal{A}_{\nu}(k), \\
\epsilon \rightarrow+0 .
\end{gathered}
$$


Здесь $\chi^{\nu \lambda}(k, p)=(p k) g^{\nu \lambda}-p^{\nu} k^{\lambda}$, и для простоты записи опушен значок Т у калибровочного поля. Подставляя (3.1) в фурье-образ соотношения (2.15), получаем известньй вид линейной по полю аппроксимации тока

$$
j^{\mathrm{T}(1) \mu}(k)=\Pi^{\mu \nu}(k) A_{\nu}(k),
$$

где

$$
\Pi^{\mu \nu}(k)=g^{2} \int d^{4} p \frac{p^{\mu}\left(p^{\nu}\left(k \partial_{p}\right)-(k p) \partial_{p}^{\nu}\right) \mathcal{N}_{\mathrm{eq}}}{p k+i p_{0} \epsilon}
$$

- высокотемпературный поляризационный тензор и

$$
\mathcal{N}_{\mathrm{eq}}=\frac{1}{2}\left(f_{\mathrm{q}}^{0}+f_{\overline{\mathrm{q}}}^{0}\right)+N_{\mathrm{c}} f_{\mathrm{g}}^{0} .
$$

Перепишем уравнение (2.16) в импульсном пространстве. Учитывая (3.2), находим

$$
\begin{aligned}
{\left[k^{2} g^{\mu \nu}\right.} & \left.-\left(1+\xi^{-1}\right) k^{\mu} k^{\nu}-\Pi^{\mu \nu}(k)\right] A_{\nu}^{b}(k)=j_{\mathrm{NL}}^{\mathrm{T} b \mu}(k)+ \\
& +f^{b c d} \int S_{k, k_{1}, k_{2}}^{\mathrm{I}) \mu \nu \lambda}\left(A_{\nu}^{c}\left(k_{1}\right) A_{\lambda}^{d}\left(k_{2}\right)-\left\langle A_{\nu}^{c}\left(k_{1}\right) A_{\lambda}^{d}\left(k_{2}\right)\right\rangle\right) \delta\left(k-k_{1}-k_{2}\right) d k_{1} d k_{2}+ \\
& +f^{b c f} f^{f d e} \int \Sigma_{k, k_{1}, k_{2}, k_{3}}^{\mu \nu \lambda \sigma}\left(A_{\nu}^{c}\left(k_{1}\right) A_{\lambda}^{d}\left(k_{2}\right) A_{\sigma}^{e}\left(k_{3}\right)-\left\langle A_{\nu}^{c}\left(k_{1}\right) A_{\lambda}^{d}\left(k_{2}\right) A_{\sigma}^{e}\left(k_{3}\right)\right\rangle\right) \times \\
& \times \delta\left(k-k_{1}-k_{2}-k_{3}\right) d k_{1} d k_{2} d k_{3}
\end{aligned}
$$

где

$$
S_{k, k_{1}, k_{2}}^{(\mathrm{I}) \mu \nu \lambda}=-i g\left(k^{\nu} g^{\mu \lambda}+k_{2}^{\nu} g^{\mu \lambda}-k_{2}^{\mu} g^{\nu \lambda}\right), \quad \Sigma_{k, k_{1}, k_{2}, k_{3}}^{\mu \nu \lambda \sigma}=g^{2} g^{\nu \lambda} g^{\mu \sigma} .
$$

Умножая уравнение (3.3) на комплексно-сопряженную амплитуду $A_{\mu}^{* a}\left(k^{\prime}\right)$, после усреднения получим

$$
\begin{aligned}
{\left[k^{2} g^{\mu \nu}\right.} & \left.-\left(1+\xi^{-1}\right) k^{\mu} k^{\nu}-\Pi^{\mu \nu}(k)\right]\left\langle A_{\mu}^{* a}\left(k^{\prime}\right) A_{\nu}^{b}(k)\right\rangle=\left\langle A_{\mu}^{* a}\left(k^{\prime}\right) j_{\mathrm{NL}}^{\mathrm{Tb} \mu}(k)\right\rangle+ \\
& +f^{b c d} \int S_{k, k_{1}, k_{2}}^{\mathrm{I}) \mu \nu \lambda}\left\langle A_{\mu}^{* a}\left(k^{\prime}\right) A_{\nu}^{c}\left(k_{1}\right) A_{\lambda}^{d}\left(k_{2}\right)\right\rangle \delta\left(k-k_{1}-k_{2}\right) d k_{1} d k_{2}+ \\
& +f^{b c f} f^{f d e} \int \Sigma_{k, k_{1}, k_{2}, k_{3}}^{\mu \nu \lambda \sigma}\left\langle A_{\mu}^{* a}\left(k^{\prime}\right) A_{\nu}^{c}\left(k_{1}\right) A_{\lambda}^{d}\left(k_{2}\right) A_{\sigma}^{e}\left(k_{3}\right)\right\rangle \times \\
& \times \delta\left(k-k_{1}-k_{2}-k_{3}\right) d k_{1} d k_{2} d k_{3} .
\end{aligned}
$$

Введем корреляционную функцию случайных колебаний

$$
I_{\mu \nu}^{a b}\left(k^{\prime}, k\right)=\left\langle A_{\mu}^{* a}\left(k^{\prime}\right) A_{\nu}^{b}(k)\right\rangle .
$$

В условиях стационарности и однородности КГП, т.е. когда корреляционная функция (3.6) в координатном представлении зависит только от разности координат и времени $\Delta X=X^{\prime}-X$, имеем

$$
I_{\mu \nu}^{a b}\left(k^{\prime}, k\right)=I_{\mu \nu}^{a b}\left(k^{\prime}\right) \delta\left(k^{\prime}-k\right) .
$$


В силу эффектов нелинейного взаимодействия волн и частиц состояние КГП становится слабонеоднородным и слабонестационарным. Зависимость функции $I_{\mu \nu}^{a b}$ от $k^{\prime}-k=\Delta k$ перестает быть $\delta$-образной.

Введем $I_{\mu \nu}^{a b}\left(k^{\prime}, k\right)=I_{\mu \nu}^{a b}(k, \Delta k)$, где $|\Delta k / k| \ll 1$, и корреляционную функцию, медленно зависящую от $x$,

$$
I_{\mu \nu}^{a b}(k, x)=\int I_{\mu \nu}^{a b}(k, \Delta k) e^{-i \Delta k x} d \Delta k
$$

В уравнении (3.5) сделаем замену $k \leftrightarrow k^{\prime}, a \leftrightarrow b$, комплексно сопряжем и вычтем полученное уравнение из (3.5), предварительно разложив поляризационный тензор на эрмитову и антиэрмитову части,

$$
\Pi^{\nu \sigma}(k)=\Pi^{\mathrm{H} \nu \sigma}(k)+\Pi^{\mathrm{A} \nu \sigma}(k), \quad \Pi^{\mathrm{H} \nu \sigma}(k)=\Pi^{* \mathrm{H} \sigma \nu}(k), \quad \Pi^{\mathrm{A} \nu \sigma}(k)=-\Pi^{* \mathrm{~A} \sigma \nu}(k) .
$$

Мы считаем, что антиэрмитова часть $\Pi^{\mathrm{A}}$ мала по сравнению с эрмитовой частью $\Pi^{\mathrm{H}}$ и является величиной того же порядка малости, что и нелинейные члены в правой части. Поэтому можно положить $\Pi^{\mathrm{A} \nu \sigma}(k) \simeq \Pi^{\mathrm{A} \nu \sigma}\left(k^{\prime}\right)$ и перенести член с $\Pi^{\mathrm{A}}$ в правую часть уравнения (3.5). Оставшиеся слагаемые в левой части разлагаем по $\Delta k$ до первого порядка малости. Умножая полученное таким образом уравнение на $e^{-i \Delta k x}$ и интегрируя по $\Delta k$, окончательно находим

$$
\begin{array}{rl}
\frac{\partial}{\partial k_{\lambda}}\left[k^{2} g^{\mu \nu}-\left(1+\xi^{-1}\right) k^{\mu} k^{\nu}-\Pi^{\mathrm{H} \mu \nu}(k)\right] \frac{\partial I_{\mu \nu}^{a b}}{\partial x^{\lambda}}= \\
=2 & i \Pi^{\mathrm{A} \mu \nu} I_{\mu \nu}^{a b}-i \int d k^{\prime}\left\{\left\langle A_{\mu}^{* a}\left(k^{\prime}\right) j_{\mathrm{NL}}^{\mathrm{T} b \mu}(k)\right\rangle-\left\langle A_{\mu}^{b}(k) j_{\mathrm{NL}}^{* \mathrm{~T} a \mu}\left(k^{\prime}\right)\right\rangle\right\}- \\
& -i\left\{f^{b c d} \int d k^{\prime} d k_{1} d k_{2} S_{k, k_{1}, k_{2}}^{(\mathrm{I}) \mu \nu \lambda}\left\langle A_{\mu}^{* a}\left(k^{\prime}\right) A_{\nu}^{\mathrm{c}}\left(k_{1}\right) A_{\lambda}^{d}\left(k_{2}\right)\right\rangle \delta\left(k-k_{1}-k_{2}\right)-\right. \\
& \left.-f^{a c d} \int d k^{\prime} d k_{1} d k_{2} S_{k^{\prime}, k_{1}, k_{2}}^{*(\mathrm{I}) \mu \nu \lambda}\left\langle A_{\mu}^{b}(k) A_{\nu}^{* c}\left(k_{1}\right) A_{\lambda}^{* d}\left(k_{2}\right)\right\rangle \delta\left(k^{\prime}-k_{1}-k_{2}\right)\right\}- \\
& -i\left\{f^{b c f} f^{f d e} \int \Sigma_{k, k_{1}, k_{2}, k_{3}}^{\mu \nu \lambda \sigma}\left\langle A_{\mu}^{* a}\left(k^{\prime}\right) A_{\nu}^{c}\left(k_{1}\right) A_{\lambda}^{d}\left(k_{2}\right) A_{\sigma}^{e}\left(k_{3}\right)\right\rangle \times\right. \\
& \times \delta\left(k-k_{1}-k_{2}-k_{3}\right) d k^{\prime} d k_{1} d k_{2} d k_{3}- \\
& -f^{a c f} f^{f d e} \int \Sigma_{k^{\prime}, k_{1}, k_{2}, k_{3}}^{* \mu \nu \lambda \sigma}\left\langle A_{\mu}^{b}(k) A_{\nu}^{* c}\left(k_{1}\right) A_{\lambda}^{* d}\left(k_{2}\right) A_{\sigma}^{* e}\left(k_{3}\right)\right\rangle \times \\
& \left.\times \delta\left(k^{\prime}-k_{1}-k_{2}-k_{3}\right) d k^{\prime} d k_{1} d k_{2} d k_{3}\right\}
\end{array}
$$

где $j_{\mathrm{NL}}^{\mathrm{T} a \mu}(k)=j^{\mathrm{T}(2) a \mu}(k)+j^{\mathrm{T}(3) a \mu}(k)$.

Сделаем несколько замечаний относительно полученного уравнения (3.8). Входящий в правую часть член с $\Pi^{\mathrm{A}}$ соответствует линейному затуханию колебаний за счет обратного эффекта Черенкова (затухание Ландау). Однако, как было показано в работе [21], линейное затухание Ландау для волн в КГП отсутствует и поэтому данный член 
можно положить равным нулю ${ }^{1)}$. Члены с $S^{(\mathrm{I})}$ и $\Sigma$ связаны с самодействием среднего поля. При учете слабой корреляции фаз случайных колебаний $A_{\mu}^{a}$, как это мы увидим ниже, члены с $S^{(\mathrm{I})}$ дадут вклад в процессы нелинейного взаимодействия волн со средой. Члены с $\Sigma$ уже имеют необходимый порядок точности и поэтому войдут в кинетическое уравнение для волн неизменными. Вследствие того что нас интересует изменение спектральной функции $I_{\mu \nu}^{a b}$ только за счет эффектов, связанных со средой, эти члены самодействия в (3.8) следует отбросить.

\section{4. ВТОРОЕ И ТРЕТЬЕ ПРИБЛИЖЕНИЯ ЦВЕТНОГО ТОКА}

Перейдем теперь к вычислению нелинейных поправок к току, входяших в правую часть уравнения (3.8) для спектральной плотности колебаний. Рассмотрим второй по-

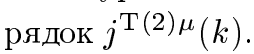

Сначала вычислим $f_{\mathrm{q}, \overline{\mathrm{q}}}^{\mathrm{T}(2)}$. Делая преобразование Фурье уравнения (2.12) и подставляя в полученное выражение найденные выше функции $f_{\mathrm{q}, \overline{\mathrm{q}}}^{\mathrm{T}(1)}(3.1)$, находим

$$
\begin{aligned}
f_{\mathrm{q}, \overline{\mathrm{q}}}^{\mathrm{T}(2)}= & \mp g^{2} \frac{\left[t^{b}, t^{c}\right] p^{\nu} p^{\lambda}}{p k+i p_{0} \epsilon} \int \frac{\left(k_{2} \partial_{p} f_{\mathrm{q}, \overline{\mathrm{q}}}^{0}\right)}{p k_{2}+i p_{0} \epsilon}\left(A_{\nu}^{b}\left(k_{1}\right) A_{\lambda}^{c}\left(k_{2}\right)-\left\langle A_{\nu}^{b}\left(k_{1}\right) A_{\lambda}^{c}\left(k_{2}\right)\right\rangle\right) \times \\
& \times \delta\left(k-k_{1}-k_{2}\right) d k_{1} d k_{2}+ \\
& +\frac{g^{2}}{2} \frac{\left\{t^{b}, t^{c}\right\}}{p k+i p_{0} \epsilon} \int \chi^{\nu \lambda}\left(k_{1}, p\right) \frac{\partial}{\partial p^{\lambda}}\left(\frac{\chi^{\sigma \rho}\left(k_{2}, p\right)}{p k_{2}+i p_{0} \epsilon} \frac{\partial f_{\mathrm{q}, \overline{\mathrm{q}}}^{0}}{\partial p^{\rho}}\right) \times \\
& \times\left(A_{\nu}^{b}\left(k_{1}\right) A_{\sigma}^{c}\left(k_{2}\right)-\left\langle A_{\nu}^{b}\left(k_{1}\right) A_{\sigma}^{c}\left(k_{2}\right)\right\rangle\right) \delta\left(k-k_{1}-k_{2}\right) d k_{1} d k_{2} .
\end{aligned}
$$

Выражение для $f_{\mathrm{g}}^{\mathrm{T}(2)}(k, p)$ получается из выражения (4.1) при выборе верхнего знака и замене $f_{\mathrm{q}}^{0} \rightarrow f_{\mathrm{g}}^{0}, t^{a} \rightarrow T^{a}$. Подставляя вычисленные выражения $f_{\mathrm{s}}^{\mathrm{T}(2)}, \mathrm{s}=\mathrm{q}, \overline{\mathrm{q}}, \mathrm{g}$, в (2.15) (при $n=2$ ), находим искомую поправку тока

$$
\begin{aligned}
j^{\mathrm{T}(2) a \mu}(k)= & -i g^{3} f^{a b c} \int d^{4} p \frac{p^{\mu} p^{\nu} p^{\lambda}}{p k+i p_{0} \epsilon} \frac{\left(k_{2} \partial_{p} \mathcal{N}_{\mathrm{eq}}\right)}{p k_{2}+i p_{0} \epsilon} \times \\
& \times\left(A_{\nu}^{b}\left(k_{1}\right) A_{\lambda}^{c}\left(k_{2}\right)-\left\langle A_{\nu}^{b}\left(k_{1}\right) A_{\lambda}^{c}\left(k_{2}\right)\right\rangle\right) \delta\left(k-k_{1}-k_{2}\right) d k_{1} d k_{2}+ \\
& +\frac{g^{3}}{4} d^{a b c} \int d^{4} p \frac{p^{\mu} \chi^{\nu \lambda}\left(k_{1}, p\right)}{p k+i p_{0} \epsilon} \frac{\partial}{\partial p^{\lambda}}\left(\frac{\chi^{\sigma \rho}\left(k_{2}, p\right)}{p k_{2}+i p_{0} \epsilon} \frac{\partial\left(f_{\mathrm{q}}^{0}-f_{\overline{\mathrm{q}}}^{0}\right)}{\partial p^{\rho}}\right) \times \\
& \times\left(A_{\nu}^{b}\left(k_{1}\right) A_{\sigma}^{c}\left(k_{2}\right)-\left\langle A_{\nu}^{b}\left(k_{1}\right) A_{\sigma}^{c}\left(k_{2}\right)\right\rangle\right) \delta\left(k-k_{1}-k_{2}\right) d k_{1} d k_{2} .
\end{aligned}
$$

Вклад глюонов в выражение при симметричной структурной константе $d^{a b c}$ здесь выпадает. Это связано с тем, что при вычислении следов от антикоммуторов мы имеем $\operatorname{Sp} t^{a}\left\{t^{b}, t^{c}\right\}=d^{a b c} / 2$ для кварков и антикварков и $\operatorname{Tr} T^{a}\left\{T^{b}, T^{c}\right\}=0$ для глюонов.

\footnotetext{
1) Отметим, что отсутствие линейного затухания Ландау в ультрарелятивистском (безмассовом) приближении было впервые показано Силиным для “горячей” электронной плазмы [15].
} 
Можно в некотором смысле восстановить симметрию вкладов, если заметить, что, кроме обычного глюонного тока $j_{\mathrm{g}}^{\mu}(x)=g t^{a} \int d^{4} p p^{\mu} \operatorname{Tr}\left(T^{a} f_{\mathrm{g}}(x, p)\right)$, кинетическое уравнение для глюонов допускает еше одну ковариантно сохраняющуюся величину

$$
\lambda g t^{a} \int d^{4} p \operatorname{Tr}\left(\mathcal{P}^{a} f_{\mathrm{g}}(x, p)\right)
$$

где $\left(\mathcal{P}^{a}\right)^{b c}=d^{a b c}$ и $\lambda$ - некоторая произвольная постоянная. Ковариантная непрерывность (4.3) вытекает из тождества $\left[\mathcal{P}^{a}, T^{b}\right]=i f^{a b c} \mathcal{P}^{c}$. Добавка (4.3) к (2.3) дает вклады только в нелинейные поправки тока. Прибавляя (4.3) ко второй итерации тока (2.15) и учитывая равенство

$$
\operatorname{Sp} \mathcal{P}^{a}\left\{T^{b}, T^{c}\right\}=N_{\mathrm{c}} d^{a b c}
$$

вместо (4.2) получим более обшее выражение для $j^{\mathrm{T}(2)}$ :

$$
\begin{aligned}
& j^{\mathrm{T}(2) a \mu}(k)=\int S_{k, k_{1}, k_{2}}^{a b c \mu \nu \lambda}\left(A_{\nu}^{b}\left(k_{1}\right) A_{\lambda}^{c}\left(k_{2}\right)-\left\langle A_{\nu}^{b}\left(k_{1}\right) A_{\lambda}^{c}\left(k_{2}\right)\right\rangle\right) \delta\left(k-k_{1}-k_{2}\right) d k_{1} d k_{2} \\
& \text { здесь } S_{k, k_{1}, k_{2}}^{a b c \mu \nu \lambda}=f^{a b c} S_{k, k_{1}, k_{2}}^{(\mathrm{II}) \mu \nu \lambda}+d^{a b c} S_{k, k_{1}, k_{2}}^{(\mathrm{III}) \mu \nu \lambda}, \\
& S_{k, k_{1}, k_{2}}^{(\mathrm{II}) \mu \lambda}=-i g^{3} \int d^{4} p \frac{p^{\mu} p^{\nu} p^{\lambda}}{p k+i p_{0} \epsilon} \frac{k_{2} \partial_{p} \mathcal{N}_{\mathrm{eq}}}{p k_{2}+i p_{0} \epsilon}, \\
& S_{k, k_{1}, k_{2}}^{(\mathrm{III}) \mu \nu \lambda}=\frac{g^{3}}{2} \int d^{4} p \frac{p^{\mu} \chi^{\nu \sigma}\left(k_{1}, p\right)}{p k+i p_{0} \epsilon} \frac{\partial}{\partial p^{\sigma}}\left(\frac{\chi^{\lambda \rho}\left(k_{2}, p\right)}{p k_{2}+i p_{0} \epsilon} \frac{\partial \mathcal{M}_{\mathrm{eq}}}{\partial p^{\rho}}\right),
\end{aligned}
$$

где

$$
\mathcal{M}_{\mathrm{eq}}=\frac{1}{2}\left(f_{\mathrm{q}}^{0}-f_{\overline{\mathrm{q}}}^{0}\right)+\lambda N_{\mathrm{c}} f_{\mathrm{g}}^{0}
$$

Тензорная структура $S_{k, k_{1}, k_{2}}^{(\mathrm{III}) \mu \nu}$ в точности совпадает с соответствующим выражением при вычислении $j^{\mathrm{T}(2) \mu}$ в абелевой плазме [27], и поэтому часть тока с $d^{a b c}$ имеет смысл абелевой части цветного тока $j^{\mathrm{T}(2) a \mu}$. Член с $S_{k, k_{1}, k_{2}}^{(\mathrm{II}) \mu \nu \lambda}$ является чисто неабелевым, т.е. не имеющим аналога в обычной плазме.

Оценим порядки величин $S^{(\mathrm{II})}$ и $S^{(\mathrm{III})}$. Следуя обычной терминологии [1], назовем энергию или импульс мягкими, если они имеют порядок $g T$, и жесткими, если они порядка $T$. Будем считать, как и в работе [18], что коллективные возбуждения переносят мягкий импульс, т.е. $k \sim g T$, в то время как частицы плазмы имеют типично жесткие энергии $p \sim T$. В координатном представлении первое из условий означает, что амплитуда колебаний $A_{\mu}^{a}(X)$ и функции распределения жестких частиц $f_{\mathrm{s}}(X, p), \mathrm{s}=\mathrm{q}, \overline{\mathrm{q}}, \mathrm{g}$, меняются на шкале $X \sim 1 / g T$. Учитывая это, будем иметь для $S^{(\mathrm{II})}$ оценку

$$
S_{k, k_{1}, k_{2}}^{(\mathrm{II}) \mu \nu \lambda} \sim g^{2} T
$$

Здесь учтено, что в силу определений $(2.17) \mathcal{N}_{\mathrm{eq}} \sim 1 / T^{2}$. 
В выражении (4.6) интеграл по энергии с глюонной функцией распределения расходится на нижнем пределе. Мы регуляризуем его аналогично работе [20], обрезав на нижнем пределе на величине порядка $g T$, и оставим только лидируюшие по $g$ члены. Такое обрезание имеет смысл, т.к. область интегрирования в (4.5) и (4.6) должна быть ограничена жесткими значениями импульса. Тогда часть, связанная с глюонной функцией распределения в (4.6), имеет порядок $g^{2} T(g \ln g)$, а оставшаяся часть, связанная с кварковой и антикварковой функциями распределений, имеет порядок $g^{3} T$, т.е.

$$
S_{k, k_{1}, k_{2}}^{(\mathrm{III}) \mu \nu \lambda} \sim g^{2} T(g \ln g)+g^{3} T .
$$

Следовательно тензор $S^{(\mathrm{II})}$, который является чисто неабелевым, имеет более низкий порядок по константе взаимодействия, чем тензор $S^{(\mathrm{III})}$, имеющий аналог в абелевой плазме. Этот факт впервые отмечен в работе [20].

Выражение для цветного тока третьего порядка по турбулентному полю определяется с помощью рассуждений, аналогичных предыдущим. Делая преобразование Фурье уравнения (2.13), учитывая (3.1), (4.1) и принимая во внимание равенства

$$
\begin{gathered}
\operatorname{Tr}\left(\left\{T^{a}, T^{b}\right\}\left\{T^{d}, T^{e}\right\}\right)=N_{\mathrm{c}} d^{a b c} d^{c d e}+4 \delta^{a b} \delta^{e d}+2 \delta^{a d} \delta^{e b}+2 \delta^{a e} \delta^{b d}, \\
\operatorname{Tr}\left(\left\{\mathcal{P}^{a}, T^{b}\right\}\left\{T^{d}, T^{e}\right\}\right)=0,
\end{gathered}
$$

после несколько громоздких преобразований находим искомый вид функции $j^{\mathrm{T}(3) a \mu}$,

$$
\begin{aligned}
& j^{\mathrm{T}(3) a \mu}(k)=\int \Sigma_{k, k_{1}, k_{2}, k_{3}}^{a b d e \mu \nu \lambda \sigma}\left(A_{\nu}^{b}\left(k_{3}\right) A_{\lambda}^{d}\left(k_{1}\right) A_{\sigma}^{e}\left(k_{2}\right)-A_{\nu}^{b}\left(k_{3}\right)\left\langle A_{\lambda}^{d}\left(k_{1}\right) A_{\sigma}^{e}\left(k_{2}\right)\right\rangle-\right. \\
& \left.\quad-\left\langle A_{\nu}^{b}\left(k_{3}\right) A_{\lambda}^{d}\left(k_{1}\right) A_{\sigma}^{e}\left(k_{2}\right)\right\rangle\right) \delta\left(k-k_{1}-k_{2}-k_{3}\right) d k_{1} d k_{2} d k_{3}+ \\
& \quad+d^{a b c} f^{c d e} \int R_{k, k_{1}, k_{2}, k_{3}}^{\mu \nu \lambda \sigma}\left(A_{\nu}^{b}\left(k_{3}\right) A_{\lambda}^{d}\left(k_{1}\right) A_{\sigma}^{e}\left(k_{2}\right)-\left\langle A_{\nu}^{b}\left(k_{3}\right) A_{\lambda}^{d}\left(k_{1}\right) A_{\sigma}^{e}\left(k_{2}\right)\right\rangle\right) \times \\
& \quad \times \delta\left(k-k_{1}-k_{2}-k_{3}\right) d k_{1} d k_{2} d k_{3} .
\end{aligned}
$$

Здесь

$$
\begin{aligned}
\Sigma_{k, k_{1}, k_{2}, k_{3}}^{a b d e \mu \nu \lambda \sigma}= & f^{a b c} f^{c d e} \Sigma_{k, k_{1}, k_{2}, k_{3}}^{(\mathrm{I}) \mu \nu \lambda \sigma}+f^{a b c} d^{c d e} \Sigma_{k, k_{1}, k_{2}, k_{3}}^{(\mathrm{II}) \mu \nu \lambda \sigma}+ \\
& +\delta^{a b} \delta^{d e} \Sigma_{k, k_{1}, k_{2}, k_{3}}^{(\mathrm{III}) \mu \nu \lambda \sigma}+d^{a b c} f^{c d e} \Sigma_{k, k_{1}, k_{2}, k_{3}}^{(\mathrm{IV}) \mu \nu \lambda \sigma}+ \\
& +d^{a b c} d^{c d e} \Sigma_{k, k_{1}, k_{2}, k_{3}}^{(\mathrm{V}) \mu \nu \lambda \sigma}+\left(\delta^{a b} \delta^{d e}+\delta^{a d} \delta^{b e}+\delta^{a e} \delta^{d b}\right) \Sigma_{k, k_{1}, k_{2}, k_{3}}^{(\mathrm{VI}) \mu \nu \lambda \sigma} \\
\Sigma_{k, k_{1}, k_{2}, k_{3}}^{(\mathrm{I}) \mu \nu \lambda \sigma}= & -g^{4} \int d^{4} p \frac{p^{\mu} p^{\nu} p^{\lambda} p^{\sigma}}{p k+i p_{0} \epsilon} \frac{1}{p\left(k_{1}+k_{2}\right)+i p_{0} \epsilon} \frac{k_{2} \partial_{p} \mathcal{N}_{\mathrm{eq}}}{p k_{2}+i p_{0} \epsilon}
\end{aligned}
$$

$$
\begin{aligned}
\Sigma_{k, k_{1}, k_{2}, k_{3}}^{(\mathrm{III}) \mu \nu \lambda \sigma}= & -\frac{g^{4}}{2 N_{\mathrm{c}}} \int d^{4} p \frac{p^{\mu} \chi^{\nu \tau}\left(k_{3}, p\right)}{p k+i p_{0} \epsilon} \times \\
& \times \frac{\partial}{\partial p^{\tau}}\left(\frac{\chi^{\lambda \alpha}\left(k_{1}, p\right)}{p\left(k_{1}+k_{2}\right)+i p_{0} \epsilon} \frac{\partial}{\partial p^{\alpha}}\left(\frac{\chi^{\sigma \rho}\left(k_{2}, p\right)}{p k_{2}+i p_{0} \epsilon} \frac{\partial \mathcal{N}_{\mathrm{eq}}}{\partial p^{\rho}}\right)\right)
\end{aligned}
$$


И

$$
\Sigma_{k, k_{1}, k_{2}, k_{3}}^{(\mathrm{V}) \mu \nu \lambda \sigma}=\frac{N_{\mathrm{c}}}{2} \Sigma_{k, k_{1}, k_{2}, k_{3}}^{(\mathrm{III}) \mu \nu \lambda \sigma}
$$

Выражение для $\Sigma^{(\mathrm{VI})}$ получается из (4.11) исключением кваркового и антикваркового вкладов. Наличие члена с $\Sigma^{(\mathrm{VI})}$ является отражением более сложной цветовой структуры глюонного кинетического уравнения в сравнении с кварковым и антикварковым, которая проявляется здесь в появлении дополнительных членов в (4.8) по сравнению с уравнением

$$
\operatorname{Sp}\left(\left\{t^{a}, t^{b}\right\}\left\{t^{d}, t^{e}\right\}\right)=\frac{1}{2} d^{a b c} d^{c d e}+\frac{1}{N_{\mathrm{c}}} \delta^{a b} \delta^{d e} .
$$

Члены с $\Sigma^{(\mathrm{II})}, \Sigma^{(\mathrm{IV})}$ и $R$ представляют собой интерференцию абелевых и неабелевых вкладов. В случае изотропной, однородной и бесцветной плазмы корреляционная функция (3.6) пропорциональна единичной матрице в цветовом пространстве, т.е. плазменные колебания являются вырожденными в цветовом отношении. Это приводит к тому, что коэффициенты перед этими интерференционными членами, несущие цветные индексы, обрашаются в нуль в кинетическом уравнении для волн, и поэтому их явный вид здесь не приводится.

В конце этого раздела сделаем оценки порядков величин $\Sigma^{(\mathrm{I})}$ и $\Sigma^{(\mathrm{III})}$. Из выражения (4.10) следует, что

$$
\Sigma_{k, k_{1}, k_{2}, k_{3}}^{(\mathrm{I}) \mu \nu \lambda \sigma} \sim g^{2} .
$$

Обрезая, как и в предыдушем разделе, предел интегрирования для глюонной функции распределения, получаем

$$
\Sigma^{(\mathrm{III})} \sim \Sigma^{(\mathrm{V})} \sim g^{3}+g^{4}, \quad \Sigma^{(\mathrm{VI})} \sim g^{3}
$$

Таким образом, чисто неабелев вклад $\Sigma^{(\mathrm{I})}$ является величиной более низкого порядка по $g$, чем абелевы вклады $\Sigma^{(\mathrm{III})}, \Sigma^{(\mathrm{V})}$ и $\Sigma^{(\mathrm{VI})}$.

\section{5. КИНЕТИЧЕСКОЕ УРАВНЕНИЕ ДЛЯ ПРОДОЛЬНЫХ ВОЛН}

Вернемся теперь к исходному уравнению для волн (3.8). Подставим в него вычисленные нелинейные поправки индуцированного полем тока (4.4) и (4.9). В силу слабой нелинейности фазы колебаний поля слабо скоррелированы между собой. Поэтому среднее значение произведения четырех случайных величин можно приближенно разбить на произведение средних значений двух полей. Для среднего трех полей такое разбиение дает нуль, и поэтому необходимо учитывать слабую корреляцию полей. Для этого воспользуемся уравнением поля (3.3), учитывая в правой части лишь члены второго порядка по $A$,

$$
\begin{aligned}
& {\left[k^{2} g^{\mu \nu}-\left(1+\xi^{-1}\right) k^{\mu} k^{\nu}-\Pi^{\mu \nu}(k)\right] A_{\nu}^{a}(k)=} \\
& \quad=\int S_{k, k_{1}, k_{2}}^{a b c \mu \nu \lambda}\left(A_{\nu}^{b}\left(k_{1}\right) A_{\lambda}^{c}\left(k_{2}\right)-\left\langle A_{\nu}^{b}\left(k_{1}\right) A_{\lambda}^{c}\left(k_{2}\right)\right\rangle\right) \delta\left(k-k_{1}-k_{2}\right) d k_{1} d k_{2} .
\end{aligned}
$$


Приближенное решение этого уравнения имеет вид

$$
\begin{aligned}
A_{\mu}^{a}(k)= & A_{\mu}^{(0) a}(k)-\mathcal{D}_{\mu \nu}(k) \int S_{k, k_{1}, k_{2}}^{a b c \nu \lambda \sigma}\left(A_{\lambda}^{(0) b}\left(k_{1}\right) A_{\sigma}^{(0) c}\left(k_{2}\right)-\right. \\
& \left.-\left\langle A_{\lambda}^{(0) b}\left(k_{1}\right) A_{\sigma}^{(0) c}\left(k_{2}\right)\right\rangle\right) \delta\left(k-k_{1}-k_{2}\right) d k_{1} d k_{2},
\end{aligned}
$$

где $A_{\mu}^{(0) a}(k)$ - решение однородного уравнения (5.1), отвечаюшее невзаимодействующим полям, а фуннция

$$
\mathcal{D}_{\mu \nu}(k)=-\left[k^{2} g_{\mu \nu}-\left(1+\xi^{-1}\right) k_{\mu} k_{\nu}-\Pi_{\mu \nu}(k)\right]^{-1}
$$

представляет собой модифицированный эффектами среды глюонный (запаздывающий) пропагатор.

Подставим теперь решение (5.2) в корреляторы трех полей, входяшие в уравнение (3.8). При этом следует уточнить каждое из слагаемых, входящее в эти корреляторы. В корреляционных функциях от четырех амплитуд в пределах принятой точности можно не делать различия между полями $A$ и $A^{(0)}$.

Уравнение (3.8) принимает в результате вид

$$
\begin{aligned}
\frac{\partial}{\partial k_{\lambda}} & {\left[k^{2} g^{\mu \nu}-\left(1+\xi^{-1}\right) k^{\mu} k^{\nu}-\Pi^{\mathrm{H} \mu \nu}(k)\right] \frac{\partial I_{\mu \nu}^{a b}}{\partial x^{\lambda}}=} \\
= & -i \int d k^{\prime} d k_{1} d k_{2} d k_{3}\left\{f^{b c f} f^{f d e} \delta\left(k-k_{1}-k_{2}-k_{3}\right) \times\right. \\
& \times \widetilde{\Sigma}_{k, k_{1}, k_{2}, k_{3}}^{\mu \nu \lambda \sigma}\left(\left\langle A_{\mu}^{* a}\left(k^{\prime}\right) A_{\nu}^{c}\left(k_{3}\right) A_{\lambda}^{d}\left(k_{1}\right) A_{\sigma}^{e}\left(k_{2}\right)\right\rangle-\left\langle A_{\mu}^{* a}\left(k^{\prime}\right) A_{\nu}^{c}\left(k_{3}\right)\right\rangle\left\langle A_{\lambda}^{d}\left(k_{1}\right) A_{\sigma}^{e}\left(k_{2}\right)\right\rangle\right)- \\
& -f^{a c f} f^{f d e} \delta\left(k^{\prime}-k_{1}-k_{2}-k_{3}\right) \widetilde{\Sigma}_{k^{\prime}, k_{1}, k_{2}, k_{3}}^{* \mu \nu \lambda}\left(\left\langle A_{\mu}^{b}(k) A_{\nu}^{* c}\left(k_{3}\right) A_{\lambda}^{* d}\left(k_{1}\right) A_{\sigma}^{* e}\left(k_{2}\right)\right\rangle-\right. \\
& \left.\left.-\left\langle A_{\mu}^{b}(k) A_{\nu}^{* c}\left(k_{3}\right)\right\rangle\left\langle A_{\lambda}^{* d}\left(k_{1}\right) A_{\sigma}^{* e}\left(k_{2}\right)\right\rangle\right)\right\}+ \\
& +i f^{b c d} f^{a e f} \int d k^{\prime} \int d k_{1} d k_{2} d k_{1}^{\prime} d k_{2}^{\prime}\left(\mathcal{D}_{\rho \alpha}^{*}\left(k^{\prime}\right)-\mathcal{D}_{\alpha \rho}(k)\right) S_{k, k_{1}, k_{2}}^{\rho \mu \nu} \times \\
& \times S_{k^{\prime}, k_{1}^{\prime}, k_{2}^{\prime}}^{* \alpha \lambda \sigma}\left(\left\langle A_{\mu}^{\mathrm{c}}\left(k_{1}\right) A_{\nu}^{d}\left(k_{2}\right) A_{\lambda}^{* e}\left(k_{1}^{\prime}\right) A_{\sigma}^{* f}\left(k_{2}^{\prime}\right)\right\rangle-\left\langle A_{\mu}^{c}\left(k_{1}\right) A_{\lambda}^{d}\left(k_{2}\right)\right\rangle\left\langle A_{\lambda}^{* e}\left(k_{1}^{\prime}\right) A_{\sigma}^{* f}\left(k_{2}^{\prime}\right)\right\rangle\right) \times \\
& \times \delta\left(k-k_{1}-k_{2}\right) \delta\left(k^{\prime}-k_{1}^{\prime}-k_{2}^{\prime}\right) .
\end{aligned}
$$

Здесь мы оставили в правой части члены только ведущего порядка по $g$ и положили

$$
\begin{aligned}
S_{k, k_{1}, k_{2}}^{\mu \nu \lambda} \equiv & S_{k, k_{1}, k_{2}}^{(\mathrm{I}) \mu \nu \lambda}+S_{k, k_{1}, k_{2}}^{(\mathrm{II}) \mu \nu \lambda} \\
\widetilde{\Sigma}_{k, k_{1}, k_{2}, k_{3}}^{\mu \nu \lambda \sigma} \equiv & \Sigma_{k, k_{1}, k_{2}, k_{3}}^{(\mathrm{I}) \mu \nu \lambda \sigma}-\left(S_{k, k_{3}, k_{1}+k_{2}}^{\mu \nu \rho}-S_{k, k_{1}+k_{2}, k_{3}}^{\mu \rho \nu}\right) \times \\
& \times \mathcal{D}_{\rho \alpha}\left(k_{1}+k_{2}\right) S_{k_{1}+k_{2}, k_{1}, k_{2}}^{\alpha \lambda \sigma} .
\end{aligned}
$$

Из определения (5.3) следует, что пропагатор имеет порядок $1 /\left(g^{2} T^{2}\right)$. Принимая во внимание $(3.4),(4.7)$ и (4.12), видим, что все члены в правой части (5.4) имеют одинаковый порядок. 
Разобьем в (5.4) средние от четырех полей на парные средние по правилу

$$
\begin{aligned}
\left\langle A\left(k_{1}\right) A\left(k_{2}\right) A\left(k_{3}\right) A\left(k_{4}\right)\right\rangle= & \left\langle A\left(k_{1}\right) A\left(k_{2}\right)\right\rangle\left\langle A\left(k_{3}\right) A\left(k_{4}\right)\right\rangle+\left\langle A\left(k_{1}\right) A\left(k_{3}\right)\right\rangle\left\langle A\left(k_{2}\right) A\left(k_{4}\right)\right\rangle+ \\
& +\left\langle A\left(k_{1}\right) A\left(k_{4}\right)\right\rangle\left\langle A\left(k_{2}\right) A\left(k_{3}\right)\right\rangle .
\end{aligned}
$$

Учитывая далее, что спектральные плотности в правой части (5.4) можно считать стационарными, однородными и равными $(3.7)$, и полагая $I_{\mu \nu}^{a b}=\delta^{a b} I_{\mu \nu}$, находим вместо (5.4)

$$
\begin{aligned}
\frac{\partial}{\partial k_{\lambda}}[ & \left.k^{2} g^{\mu \nu}-\left(1+\xi^{-1}\right) k^{\mu} k^{\nu}-\Pi^{\mathrm{H} \mu \nu}(k)\right] \frac{\partial I_{\mu \nu}}{\partial x^{\lambda}}= \\
= & 2 N_{\mathrm{c}} \int d k_{1} \operatorname{Im}\left(\widetilde{\Sigma}_{k, k, k_{1},-k_{1}}^{\mu \nu \lambda \sigma}-\widetilde{\Sigma}_{k, k_{1}, k,-k_{1}}^{\mu \nu \sigma \lambda}\right) I_{\mu \lambda}(k) I_{\nu \sigma}\left(k_{1}\right)+ \\
& +N_{\mathrm{c}} \operatorname{Im}\left(\mathcal{D}_{\rho \alpha}(k)\right) \int d k_{1} d k_{2}\left(S_{k, k_{1}, k_{2}}^{\rho \mu \nu}-S_{k, k_{2}, k_{1}}^{\rho \nu \mu}\right)\left(S_{k, k_{1}, k_{2}}^{* \alpha \lambda \sigma}-S_{k, k_{2}, k_{1}}^{* \alpha \sigma \lambda}\right) \times \\
& \times I_{\mu \lambda}\left(k_{1}\right) I_{\nu \sigma}\left(k_{2}\right) \delta\left(k-k_{1}-k_{2}\right) .
\end{aligned}
$$

Как известно [28-30], в глобально равновесной КГП могут распространяться колебания трех видов: продольные, поперечные и четырехмерно-продольные, не имеющие физического смысла. В связи с этим спектральную плотность $I_{\mu \nu}(k, x)=I_{\mu \nu}$ представим в виде разложения

$$
I_{\mu \nu}=P_{\mu \nu} I_{k}^{t}+Q_{\mu \nu} I_{k}^{l}+\xi D_{\mu \nu} I_{k}^{n}, \quad I_{k}^{(t, l, n)} \equiv I^{(t, l, n)}(k, x) .
$$

Лоренц-инвариантные матрицы в (5.7) соответствуют матричному базису $[31,32]$

$$
\begin{gathered}
P_{\mu \nu}(k)=g_{\mu \nu}-D_{\mu \nu}(k)-Q_{\mu \nu}(k), \quad Q_{\mu \nu}(k)=\frac{\bar{u}_{\mu}(k) \bar{u}_{\nu}(k)}{\bar{u}^{2}(k)}, \\
C_{\mu \nu}(k)=-\frac{\bar{u}_{\mu}(k) k_{\nu}+\bar{u}_{\nu}(k) k_{\mu}}{\sqrt{-2 k^{2} \bar{u}^{2}(k)}}, \quad D_{\mu \nu}=\frac{k_{\mu} k_{\nu}}{k^{2}}, \quad \bar{u}_{\mu}(k)=k^{2} u_{\mu}-k_{\mu}(k u) .
\end{gathered}
$$

Эффективный глюонный пропагатор (5.3) можно записать в более привычном виде

$$
\mathcal{D}_{\mu \nu}(k)=-P_{\mu \nu}(k) \Delta^{t}(k)-Q_{\mu \nu}(k) \Delta^{l}(k)+\xi D_{\mu \nu}(k) \Delta^{0}(k),
$$

где

$$
\begin{gathered}
\Delta^{t, l}(k)=\frac{1}{k^{2}-\Pi^{t, l}(k)}, \quad \Pi^{t}=\frac{1}{2} \Pi^{\mu \nu} P_{\mu \nu}, \quad \Pi^{l}=\Pi^{\mu \nu} Q_{\mu \nu}, \\
\Delta^{0}(k)=\frac{1}{(\omega+i \epsilon)^{2}-\mathbf{k}^{2}}
\end{gathered}
$$

$\xi$ - калибровочный параметр. Сдвиг $i \epsilon$ введен в $\Delta^{0}(k)$ для того, чтобы обеспечить правильные аналитические свойства [33]. Отметим, что, хотя все дальнейшие вычисления 
можно проводить при произвольном значении 4-скорости плазмы $u_{\mu}$, мы, однако, для простоты будем считать плазму покоящейся, т.е. положим $u_{\mu}=(1,0,0,0)$.

Дальнейший вывод кинетического уравнения для продольных колебаний аналогичен соответствуюшим рассуждениям в теории электрон-ионной плазмы, поэтому ограничимся лишш его схематичным описанием.

Отбросим в уравнении (3.5) нелинейные члены и антиэрмитову часть тензора поляризации. Подставляя далее $\delta^{a b} Q_{\mu \nu}(k) I_{k}^{l} \delta\left(k^{\prime}-k\right)$ вместо $I_{\mu \nu}^{a b}\left(k^{\prime}, k\right)$, приходим к уравнению

$$
\operatorname{Re}\left(\varepsilon^{l}(k)\right) I_{k}^{l}=0 .
$$

Здесь мы использовали соотношение

$$
\Delta^{-1 l}(k)=k^{2} \varepsilon^{l}(k)
$$

где продольная диэлектрическая проницаемость КГП

$$
\varepsilon^{l}(k)=1+\frac{3 \omega_{\mathrm{pl}}^{2}}{\mathbf{k}^{2}}\left[1-F\left(\frac{\omega}{|\mathbf{k}|}\right)\right], \quad F(x)=\frac{x}{2}\left[\ln \left|\frac{1+x}{1-x}\right|-i \pi \theta(1-|x|)\right]
$$

и

$$
\omega_{\mathrm{pl}}^{2}=\frac{1}{18} g^{2} T^{2}\left(N_{\mathrm{f}}+2 N_{\mathrm{c}}\right)
$$

- плазменная частота. Решение этого уравнения имеет вид

$$
I_{k}^{l}=I_{\mathbf{k}}^{l} \delta\left(\omega-\omega_{\mathbf{k}}^{l}\right)+I_{-\mathbf{k}}^{l} \delta\left(\omega+\omega_{\mathbf{k}}^{l}\right), \quad \omega_{\mathbf{k}}^{l}>0
$$

где $I_{\mathbf{k}}^{l}$ - некоторая функция волнового вектора $\mathbf{k}$, а $\omega_{\mathbf{k}}^{l} \equiv \omega^{l}(\mathbf{k})$ - частота собственного продольного колебания КГП.

Уравнение, описывающее изменение спектральной плотности продольных колебаний, получается из (5.6) заменой $I_{\mu \nu} \rightarrow Q_{\mu \nu}(k) I_{k}^{l}$, где $I_{k}^{l}$ определяется в $(5.10) . \delta$-Функции в (5.10) позволяют снять интегрирование по частотам, и, таким образом, вместо (5.6) будем иметь

$$
\begin{aligned}
& \left(k^{2} \frac{\partial \operatorname{Re} \varepsilon^{l}(k)}{\partial k_{\lambda}}\right)_{\omega=\omega_{\mathbf{k}}^{l}} \frac{\partial I_{\mathbf{k}}^{l}}{\partial x^{\lambda}}=2 N_{\mathrm{c}} I_{\mathbf{k}}^{l} \int d \mathbf{k}_{1} I_{\mathbf{k}_{1}}^{l}\left(\operatorname { I m } \left[\left(\widetilde{\Sigma}_{k, k, k_{1},-k_{1}}^{\mu \nu \lambda \sigma}-\widetilde{\Sigma}_{k, k_{1}, k,-k_{1}}^{\mu \nu \sigma \lambda}\right)+\right.\right. \\
& \left.\left.\quad+\left(\widetilde{\Sigma}_{k, k,-k_{1}, k_{1}}^{\mu \nu \lambda \sigma}-\widetilde{\Sigma}_{k,-k_{1}, k, k_{1}}^{\mu \nu \sigma \lambda}\right)\right] Q_{\mu \lambda}(k) Q_{\nu \sigma}\left(k_{1}\right)\right)\left.\right|_{\omega=\omega_{\mathbf{k}}^{l}, \omega_{1}=\omega_{\mathbf{k}_{1}}^{l}}+ \\
& \quad+N_{\mathrm{c}} \int_{0}^{\infty} d \omega \int d \mathbf{k}_{1} d \mathbf{k}_{2} I_{\mathbf{k}_{1}}^{l} I_{\mathbf{k}_{2}}^{l} \times \\
& \quad \times\left.\left(G_{k, k_{1}, k_{2}}+G_{k,-k_{1}, k_{2}}+G_{k, k_{1},-k_{2}}+G_{k,-k_{1},-k_{2}}\right)\right|_{\omega_{1}=\omega_{\mathbf{k}_{1}}^{l}, \omega_{2}=\omega_{\mathbf{k}_{2}}^{l}},
\end{aligned}
$$

где

$$
\begin{aligned}
G_{k, k_{1}, k_{2}}= & \operatorname{Im}\left(\mathcal{D}_{\rho \alpha}(k)\right)\left(S_{k, k_{1}, k_{2}}^{\rho \mu \nu}-S_{k, k_{2}, k_{1}}^{\rho \nu \mu}\right) \times \\
& \times\left(S_{k, k_{1}, k_{2}}^{* \alpha \lambda \sigma}-S_{k, k_{2}, k_{1}}^{* \alpha \sigma \lambda}\right) Q_{\mu \lambda}\left(k_{1}\right) Q_{\nu \sigma}\left(k_{2}\right) \delta\left(k-k_{1}-k_{2}\right) .
\end{aligned}
$$


Рассмотрим в отдельности члены, входящие в правую часть уравнения (5.11). Интеграл с $G$-функциями, описывающий трехплазмонные процессы в КГП, равен нулю в силу нераспадного характера спектра продольных колебаний. Выражение

$$
\left.\left(\widetilde{\Sigma}_{k, k,-k_{1}, k_{1}}^{\mu \nu \lambda \sigma}-\widetilde{\Sigma}_{k,-k_{1}, k, k_{1}}^{\mu \nu \sigma \lambda}\right) Q_{\mu \lambda}(k) Q_{\nu \sigma}\left(k_{1}\right)\right|_{\omega=\omega_{\mathbf{k}}^{l}, \omega_{1}=\omega_{\mathbf{k}_{1}}^{l}}
$$

содержит в силу определения входящих в него функций факторы

$$
\frac{1}{p k+i p_{0} \epsilon}, \quad \frac{1}{p k_{1}+i p_{0} \epsilon}, \quad \frac{1}{p\left(k-k_{1}\right)+i p_{0} \epsilon} .
$$

Мнимые части первых двух из них необходимо положить равными нулю, т.к. они связаны с линейным затуханием Ландау продольных волн (которое отсутствует в КГП), и поэтому мнимая часть выражения (5.12), собственно входящая в (5.11), будет определяться соотношением

$$
\left.\operatorname{Im} \frac{1}{p\left(k-k_{1}\right)+i p_{0} \epsilon}\right|_{\omega=\omega_{\mathbf{k}}^{l}, \omega_{1}=\omega_{\mathbf{k}_{1}}^{l}}=-\frac{i \pi}{p_{0}} \delta\left(\omega_{\mathbf{k}}^{l}-\omega_{\mathbf{k}_{1}}^{l}-\mathbf{v}\left(\mathbf{k}-\mathbf{k}_{1}\right)\right) .
$$

Аргумент $\delta$-функции в правой части представляет собой закон сохранения энергии в процессе рассеяния продольной волны (плазмона) в продольную волну на частицах КГП.

Несколько более подробно рассмотрим слагаемое в выражении (5.12) (см. определение $(5.5))$ с пропагатором $\mathcal{D}_{\rho \alpha}\left(k-k_{1}\right)$. В силу разложения (5.8) этот пропагатор описывает нелинейное взаимодействие продольных волн с продольными волнами через три типа промежуточных колебаний: поперечное, продольное и нефизическое, зависяшее от калибровочного параметра. Член с

$$
\left.\left(P_{\rho \alpha}\left(k-k_{1}\right) \Delta^{t}\left(k-k_{1}\right)\right)\right|_{\omega=\omega_{\mathbf{k}}^{l}, \omega_{1}=\omega_{\mathbf{k}_{1}}^{l}}
$$

описывает, вообше говоря, два принципиально разных нелинейных процесса:

1) если $\left(\omega_{\mathbf{k}}^{l}-\omega_{\mathbf{k}_{1}}^{l}, \mathbf{k}-\mathbf{k}_{1}\right)$ является решением дисперсионного уравнения $\Delta^{t}\left(k-k_{1}\right)=0$, то этот член описывает процесс слияния двух продольных колебаний в одно поперечное;

2) в противном случае он определяет процесс нелинейного рассеяния продольных волн в продольные волны через поперечное виртуальное колебание.

Для продольных колебаний равенство $\left.\Delta^{l}\left(k-k_{1}\right)\right|_{\omega=\omega_{\mathbf{k}}^{l}, \omega_{1}=\omega_{\mathbf{k}_{1}}^{l}}=0$ не вьполняется и поэтому член

$$
\left.\left(Q_{\rho \alpha}\left(k-k_{1}\right) \Delta^{l}\left(k-k_{1}\right)\right)\right|_{\omega=\omega_{\mathbf{k}}^{l}, \omega_{1}=\omega_{\mathbf{k}_{1}}^{l}}
$$

определяет только процесс рассеяния продольных волн в продольные через продольное виртуальное колебание. Вклад нефизического промежуточного колебания

$$
\left.\left(\xi D_{\rho \alpha}\left(k-k_{1}\right) \Delta^{0}\left(k-k_{1}\right)\right)\right|_{\omega=\omega_{\mathbf{k}}^{l}, \omega_{1}=\omega_{\mathbf{k}_{1}}^{l}}
$$


будет рассмотрен отдельно в разделе 4 второй части работы.

Оставшиеся члены с $\widetilde{\Sigma}$ отличаются от рассмотренных выше знаком перед $k_{1}$ и описывают процессы одновременного излучения или поглощения частицами двух волн.

Суммируя все вышесказанное и переходя от функции $I_{\mathbf{k}}^{l}$ к функции

$$
W_{\mathbf{k}}^{l}=-\left.\left(\omega k^{2} \frac{\partial \operatorname{Re} \varepsilon^{l}(k)}{\partial \omega}\right)\right|_{\omega=\omega_{\mathbf{k}}^{l}} I_{\mathbf{k}}^{l}
$$

имеюшей физический смысл спектральной плотности энергии продольных колебаний, получаем из (5.11) искомое кинетическое уравнение для продольных волн в КГП

$$
\frac{\partial W_{\mathbf{k}}^{l}}{\partial t}+\mathbf{V}_{\mathbf{k}}^{l} \frac{\partial W_{\mathbf{k}}^{l}}{\partial \mathbf{x}}=-\hat{\gamma}\left\{\left(\frac{W_{\mathbf{k}}^{l}}{\omega_{\mathbf{k}}^{l}}\right)\right\} W_{\mathbf{k}}^{l},
$$

где

$$
\mathbf{V}_{\mathbf{k}}^{l}=\frac{\partial \omega_{\mathbf{k}}^{l}}{\partial \mathbf{k}}=-\left.\left[\left(\frac{\partial \operatorname{Re} \varepsilon^{l}(k)}{\partial \mathbf{k}}\right)\left(\frac{\partial \operatorname{Re} \varepsilon^{l}(k)}{\partial \omega}\right)^{-1}\right]\right|_{\omega=\omega_{\mathbf{k}}^{l}}
$$

- групповая скорость продольных колебаний, а выражение

$$
\begin{aligned}
\hat{\gamma}\left\{\left(\frac{W_{\mathbf{k}}^{l}}{\omega_{\mathbf{k}}^{l}}\right)\right\} \equiv \gamma^{l}(\mathbf{k})= \\
=2 N_{\mathrm{c}} \int d \mathbf{k}_{1}\left(\frac{W_{\mathbf{k}_{1}}^{l}}{\omega_{\mathbf{k}_{1}}^{l}}\right)\left[\frac{1}{k^{2} k_{1}^{2}}\left(\frac{\partial \operatorname{Re} \varepsilon^{l}(k)}{\partial \omega}\right)^{-1}\left(\frac{\partial \operatorname{Re} \varepsilon^{l}\left(k_{1}\right)}{\partial \omega_{1}}\right)^{-1} \times\right. \\
\quad \times \operatorname{Im}\left[\left(\widetilde{\Sigma}_{k, k, k_{1},-k_{1}}^{\mu \nu \lambda \sigma}-\widetilde{\Sigma}_{k, k_{1}, k,-k_{1}}^{\mu \nu \nu \lambda}\right)+\left(\widetilde{\Sigma}_{k, k,-k_{1}, k_{1}}^{\mu \nu \lambda \sigma}-\widetilde{\Sigma}_{k,-k_{1}, k, k_{1}}^{\mu \nu \sigma \lambda}\right)\right] \times \\
\left.\quad \times Q_{\mu \lambda}(k) Q_{\nu \sigma}\left(k_{1}\right)\right]\left.\right|_{\omega=\omega_{\mathbf{k}}^{l}, \omega_{1}=\omega_{\mathbf{k}_{1}}^{l}}
\end{aligned}
$$

представляет собой декремент затухания, обусловленный нелинейными эффектами.

Если ввести спектральную плотность числа продольных колебаний

$$
N_{\mathbf{k}}^{l}=\frac{W_{\mathbf{k}}^{l}}{\omega_{\mathbf{k}}^{l}}
$$

играюшую роль функции распределения числа плазмонов, то уравнение (5.14) можно представить также в виде

$$
\frac{d N_{\mathbf{k}}^{l}}{d t} \equiv \frac{\partial N_{\mathbf{k}}^{l}}{\partial t}+\mathbf{V}_{\mathbf{k}}^{l} \frac{\partial N_{\mathbf{k}}^{l}}{\partial \mathbf{x}}=-\hat{\gamma}\left\{N_{\mathbf{k}}^{l}\right\} N_{\mathbf{k}}^{l}
$$

В заключение этого раздела сделаем одно важное замечание. В данной работе мы сосредоточили свое внимание на выводе кинетического уравнения для плазмонов (5.16), 
использование которого необходимо для описания турбулентных флуктуаций коллективных возбуждений КГП. Однако нужно отметить, что в этой статье был принят ряд предположений, фактически запрешаюших возникновение турбулентного состояния КГП. Так, например, в разделе 2 мы положили регулярную часть калибровочного поля равной нулю, а регулярные части функций распределения кварков (антикварков) и глюонов равными соответственно равновесным распределениям Ферми-Дирака и Бозе-Эйнштейна (2.17), тем самым устранив сушественные причины турбулизации КГП.

Кроме того, из-за принятого нами ультрарелятивистского (безмассового) приближения в описании КГП линейное затухание Ландау отсутствует, что также запрешает возбуждение турбулентности вследствие черенковского излучения плазменных волн частицами $\mathrm{K}^{2} \Pi^{2}$. Однако все эти ограничиваюшие турбулентность предположения несущественны для вывода кинетического уравнения (5.16), которое является основным предметом данной статьи.

\section{6. НТL-ФУНКЦИИ. ТОЖДЕСТВА УОРДА}

Прежде чем перейти к вопросу о калибровочной зависимости найденного декремента нелинейного затухания Ландау (5.15), перепишем полученное выражение в терминах HTL-функций $[1,2,18]$. Это позволяет перенести методику доказательства калибровочной инвариантности декремента затухания коллективных возбуждений КГП в квантовой теории [1] на наш случай.

Представим меру интегрирования $d^{4} p$ в виде $d p^{0}|\mathbf{p}|^{2} d|\mathbf{p}| d \Omega$, где $d \Omega$ - элемент телесного угла относительно единичного вектора $\mathbf{v}=\mathbf{p} /|\mathbf{p}|$. Используя определения равновесных функций распределения (2.17) (при $\mu=0$ ) и учитывая, что

$$
\int_{-\infty}^{+\infty}|\mathbf{p}|^{2} d|\mathbf{p}| \int_{-\infty}^{+\infty} p_{0} d p_{0} \frac{d \mathcal{N}_{\mathrm{eq}}\left(p_{0}\right)}{d p_{0}}=-\frac{3}{4 \pi}\left(\frac{\omega_{\mathrm{pl}}}{g}\right)^{2}
$$

снимем интегрирование по $d p_{0}$ и $d|\mathbf{p}|$ в выражениях для $S$-функции (4.5) и $\Sigma^{(\mathrm{I})}$-фуннкции (4.10). Перепишем далее выражение (5.15) следуюшим образом:

$$
\begin{aligned}
\gamma^{l}(\mathbf{k})= & -2 g^{2} N_{\mathrm{c}} \int d \mathbf{k}_{1}\left(\frac{W_{\mathbf{k}_{1}}^{l}}{\omega_{\mathbf{k}_{1}}^{l}}\right)\left[\frac{1}{k^{2} k_{1}^{2}}\left(\frac{\partial \operatorname{Re} \varepsilon^{l}(k)}{\partial \omega}\right)^{-1}\left(\frac{\partial \operatorname{Re} \varepsilon^{l}\left(k_{1}\right)}{\partial \omega_{1}}\right)^{-1} \times\right. \\
& \times \operatorname{Im}\left\{\delta \Gamma^{\mu \nu \lambda \sigma}\left(k, k_{1},-k,-k_{1}\right)-{ }^{*} \Gamma^{\mu \nu \rho}\left(k,-k_{1},-k+k_{1}\right) \mathcal{D}_{\rho \alpha}\left(k-k_{1}\right) \times\right. \\
& \times{ }^{*} \Gamma^{\alpha \lambda \sigma}\left(k-k_{1},-k, k_{1}\right)-{ }^{*} \Gamma^{\mu \nu \rho}\left(k, k_{1},-k-k_{1}\right) \mathcal{D}_{\rho \alpha}\left(k+k_{1}\right) \times \\
& \left.\left.\times{ }^{*} \Gamma^{\alpha \lambda \sigma}\left(k+k_{1},-k,-k_{1}\right)\right\} Q_{\mu \lambda}(k) Q_{\nu \sigma}\left(k_{1}\right)\right]\left.\right|_{\omega=\omega_{\mathbf{k}}^{l}, \omega_{1}=\omega_{\mathbf{k}_{1}}^{l}},
\end{aligned}
$$

\footnotetext{
2) Здесь необходимо сделать некоторое уточнение. Может показаться, что учет конечной массы кварков (например, токового $s$-кварка, масса которого уже сравнима с температурой КГП при ее возможном образовании в экспериментах по соударению тяжелых ионов) делает разрешенным черенковский механизм возбуждения плазменной турбулентности. Однако, как показано во второй части работы [10], даже в случае учета массы кварков линейное затухание Ландау продольных цветных плазмонов отсутствует из-за наличия в плазме безмассовых глюонов.
} 
где

$$
\begin{aligned}
\delta \Gamma^{\mu \nu \lambda \sigma}\left(k, k_{1}, k_{2}, k_{3}\right)= & 3 \omega_{\mathrm{pl}}^{2} \int \frac{d \Omega}{4 \pi} \frac{v^{\mu} v^{\nu} v^{\lambda} v^{\sigma}}{v k+i \epsilon}\left[\frac{1}{v\left(k+k_{1}\right)+i \epsilon}\left(\frac{\omega_{2}}{v k_{2}-i \epsilon}-\frac{\omega_{3}}{v k_{3}-i \epsilon}\right)-\right. \\
& \left.-\frac{1}{v\left(k+k_{3}\right)+i \epsilon}\left(\frac{\omega_{1}}{v k_{1}-i \epsilon}-\frac{\omega_{2}}{v k_{2}-i \epsilon}\right)\right], \quad v^{\mu}=(1, \mathbf{v}), \quad(6.2)
\end{aligned}
$$

представляет собой HTL-поправку к голой четырехглюонной вершине $[1,2,18]$,

$$
{ }^{*} \Gamma^{\mu \nu \rho}\left(k, k_{1}, k_{2}\right) \equiv \Gamma^{\mu \nu \rho}\left(k, k_{1}, k_{2}\right)+\delta \Gamma^{\mu \nu \rho}\left(k, k_{1}, k_{2}\right)
$$

- эффективная трехглюонная вершина $[1,2,18]$, которая является суммой голой трехглюонной вершины

$$
\Gamma^{\mu \nu \rho}\left(k, k_{1}, k_{2}\right)=g^{\mu \nu}\left(k-k_{1}\right)^{\rho}+g^{\nu \rho}\left(k_{1}-k_{2}\right)^{\mu}+g^{\mu \rho}\left(k_{2}-k\right)^{\nu}
$$

и соответствуюшей HTL-поправки

$$
\delta \Gamma^{\mu \nu \rho}\left(k, k_{1}, k_{2}\right)=3 \omega_{\mathrm{pl}}^{2} \int \frac{d \Omega}{4 \pi} \frac{v^{\mu} v^{\nu} v^{\rho}}{v k+i \epsilon}\left(\frac{\omega_{2}}{v k_{2}-i \epsilon}-\frac{\omega_{1}}{v k_{1}-i \epsilon}\right) .
$$

Поляризационный тензор в этих обозначениях имеет вид

$$
\Pi^{\mu \nu}(k)=3 \omega_{\mathrm{pl}}^{2}\left(u^{\mu} u^{\nu}-\omega \int \frac{d \Omega}{4 \pi} \frac{v^{\mu} v^{\nu}}{v k+i \epsilon}\right) .
$$

Для того чтобы записать выражение (6.1), например в $A_{0}$-калибровке, достаточно сделать замену проекторов $Q_{\mu \lambda}(k)$ и $Q_{\nu \sigma}\left(k_{1}\right)$ на

$$
\begin{gathered}
\widetilde{Q}_{\mu \lambda}(k)=Q_{\mu \lambda}(k)+\frac{\sqrt{-2 k^{2} \bar{u}^{2}}}{k^{2}(k u)} C_{\mu \lambda}(k)+\frac{\bar{u}^{2}(k)}{k^{2}(k u)^{2}} D_{\mu \lambda}(k)=\frac{\tilde{u}_{\mu}(k) \tilde{u}_{\lambda}(k)}{\bar{u}^{2}(k)}, \\
\tilde{u}_{\mu}(k) \equiv \frac{k^{2}}{(k u)}\left(k_{\mu}-u_{\mu}(k u)\right)
\end{gathered}
$$

(аналогично для $\left.Q_{\nu \sigma}\left(k_{1}\right)\right)$, а пропагатор (5.8) заменить на

$$
\begin{aligned}
\widetilde{\mathcal{D}}_{\rho \alpha}(k)= & \mathcal{D}_{\rho \alpha}(k)-\left(\frac{\sqrt{-2 k^{2} \bar{u}^{2}}}{k^{2}(k u)} C_{\rho \alpha}(k)+\frac{\bar{u}^{2}(k)}{k^{2}(k u)^{2}} D_{\rho \alpha}(k)\right) \Delta^{l}(k)- \\
& -\xi D_{\rho \alpha}(k) \Delta^{0}(k)-\xi_{0} \frac{k^{2}}{(k u)^{2}} D_{\rho \alpha}(k)
\end{aligned}
$$

где $\xi_{0}$ - калибровочный параметр в $A_{0}$-калибровке.

Для доказательства калибровочной инвариантности декремента нелинейного затухания Ландау $\gamma^{l}(\mathbf{k})$ необходимо показать, что выражение, стояшее под знаком мнимой

5 Теоретическая и математическая физика, т. 124, № 1, 2000 г. 
части в (6.1), в ковариантной калибровке тождественно равно аналогичному выражению в $A_{0}$-калибровке.

Мы, однако, докажем калибровочную инвариантность более обшего выражения, которое в ковариантной калибровке имеет вид

$$
\begin{aligned}
* \widetilde{\Gamma}(k, & \left.-k_{2}, k_{1},-k_{3}\right) \equiv\left\{{ }^{*} \Gamma^{\mu \sigma \lambda \nu}\left(k,-k_{2}, k_{1},-k_{3}\right)-\mathcal{D}_{\rho \alpha}\left(-k_{1}+k_{2}\right) \times\right. \\
& \times{ }^{*} \Gamma^{\mu \nu \rho}\left(k,-k_{3}, k_{1}-k_{2}\right)^{*} \Gamma^{\alpha \lambda \sigma}\left(-k_{1}+k_{2}, k_{1},-k_{2}\right)- \\
& -\mathcal{D}_{\rho \alpha}\left(-k_{1}+k_{3}\right)^{*} \Gamma^{\mu \sigma \rho}\left(k,-k_{2}, k_{1}-k_{3}\right) \times \\
& \left.\times{ }^{*} \Gamma^{\alpha \lambda \nu}\left(-k_{1}+k_{3}, k_{1},-k_{3}\right)\right\}\left.\bar{u}_{\mu}(k) \bar{u}_{\lambda}\left(k_{1}\right) \bar{u}_{\nu}\left(k_{3}\right) \bar{u}_{\sigma}\left(k_{2}\right)\right|_{\text {on-shell }} .
\end{aligned}
$$

Здесь $k+k_{1}=k_{2}+k_{3} ;{ }^{*} \Gamma^{\mu \sigma \lambda \nu}=\Gamma^{\mu \sigma \lambda \nu}+\delta \Gamma^{\mu \sigma \lambda \nu}$ представляет собой эффективную 4-глюонную вершину, состояшую из суммы голой 4-глюонной вершины

$$
\Gamma^{\mu \sigma \lambda \nu}=2 g^{\mu \sigma} g^{\lambda \nu}-g^{\mu \nu} g^{\sigma \lambda}-g^{\mu \lambda} g^{\nu \sigma}
$$

и (4-HTL)-поправки (6.2). Выражение (6.7) переходит в выражение, стояшее под знаком мнимой части в $(6.1)$, если положить $k_{1}=-k, k_{2}=-k_{1}$ и $k_{3}=k_{1}$. Как будет нами показано в одной из следуюших работ, выражение (6.7) связано с вероятностью плазмон-плазмонного рассеяния.

Аналогичное выражение $(6.7)$ в $A_{0}$-калибровке получается заменой $\bar{u}_{\mu}(k) \rightarrow \tilde{u}_{\mu}(k)$ и пропагатора (5.8) на (6.6).

Доказательство калибровочной инвариантности основано на использовании тождеств, аналогичных эффективным тождествам Уорда в высотемпературной КХ Д $[1,2]$. Можно показать, что справедливы равенства

$$
\begin{aligned}
k_{\mu}{ }^{*} \Gamma^{\mu \nu \lambda \sigma}\left(k, k_{1}, k_{2}, k_{3}\right) & ={ }^{*} \Gamma^{\nu \lambda \sigma}\left(k_{1}, k_{2}, k+k_{3}\right)-{ }^{*} \Gamma^{\nu \lambda \sigma}\left(k+k_{1}, k_{2}, k_{3}\right), \\
k_{1 \nu}{ }^{*} \Gamma^{\mu \nu \lambda \sigma}\left(k, k_{1}, k_{2}, k_{3}\right) & ={ }^{*} \Gamma^{\mu \lambda \sigma}\left(k+k_{1}, k_{2}, k_{3}\right)-{ }^{*} \Gamma^{\mu \lambda \sigma}\left(k, k_{1}+k_{2}, k_{3}\right)
\end{aligned}
$$

(аналогичные свертки с $k_{2 \lambda}, k_{3 \sigma}$ ),

$$
k_{\mu}^{*} \Gamma^{\mu \nu \rho}\left(k, k_{1}, k_{2}\right)=\mathcal{D}^{-1 \nu \rho}\left(-k_{1}\right)-\mathcal{D}^{-1 \nu \rho}\left(-k_{2}\right)
$$

(аналогичные свертки с $k_{1 \nu}, k_{2 \rho}$ ). Здесь

$$
\mathcal{D}^{-1 \mu \nu}(k)=P^{\mu \nu}(k) \Delta^{-1 t}(k)+Q^{\mu \nu}(k) \Delta^{-1 l}(k)
$$

- обратный пропагатор, для которого справедливо соотношение

$$
\mathcal{D}_{\rho \alpha} \mathcal{D}^{-1 \alpha \lambda}(k)=\delta_{\rho}^{\lambda}-\frac{k_{\rho} k^{\lambda}}{k^{2}}
$$




\section{7. КАЛИБРОВОЧНАЯ ИНВАРИАНТНОСТЬ}

Доказательство калибровочной ивариантности сводится к последовательной свертке проекторов $Q$ и $\widetilde{Q}$ с ресуммированными 3 - и 4-глюонными вершинами и использованию тождеств Уорда (6.8), (6.9). Рассмотрим сначала член с калибровочным параметром в пропагаторе в ковариантной калибровке (5.8). В силу (6.9) имеем

$$
\begin{aligned}
& \xi D_{\rho \alpha}\left(k_{1}-k_{2}\right) \Delta^{0}\left(k_{1}-k_{2}\right)^{*} \Gamma^{\mu \nu \rho}\left(k_{1},-k_{3}, k_{1}-k_{2}\right)^{*} \Gamma^{\alpha \lambda \sigma}\left(-k_{1}+k_{2}, k_{1},-k_{2}\right)= \\
& \quad=\xi\left(\Delta^{0}\left(k_{1}-k_{2}\right)\right)^{2}\left(\mathcal{D}^{-1 \mu \sigma}(k)-\mathcal{D}^{-1 \mu \sigma}\left(k_{3}\right)\right)\left(\mathcal{D}^{-1 \lambda \nu}\left(k_{2}\right)-\mathcal{D}^{-1 \lambda \nu}\left(-k_{1}\right)\right) .
\end{aligned}
$$

Свертывая далее это выражение с $\bar{u}_{\mu}(k) \bar{u}_{\nu}\left(k_{3}\right)$, легко показать, что оно обрашается в нуль либо в силу условия массовой поверхности, либо в силу ортогональности обратного пропагатора, т.е.

$$
\left.\mathcal{D}^{-10 \nu}(k)\right|_{\omega=\omega_{\mathbf{k}}^{l}}=0, \quad k_{\mu} \mathcal{D}^{-1 \mu \nu}(k)=0 .
$$

Аналогичное утверждение справедливо и для $A_{0}$-калибровки.

В формальных вычислениях, приведенных вьше, калибровочно-зависимые части выпадают из $\gamma^{l}(\mathbf{k})$, т.к. они умножаются на факторы, представляюшие собой уравнения массовой поверхности. Однако, как было показано в работе [34], в квантовом случае эти факторы умножаются на интеграл, включаюший степенную инфракрасную расходимость, которая компенсирует в точности (в ковариантной калибровке) условия массовой поверхности. Таким образом, калибровочно-зависимая часть дает конечный вклад в глюонный декремент затухания. Данный вопрос изучался также для декремента затухания ферми-возбуждений КГП $[6,33,35,36]$.

Можно показать, что и в нашем случае интеграл, стояший перед условием массовой поверхности для $(\mathbf{k}=0)$-моды, также расходится на нижнем пределе; и, таким образом, возникает аналогичная проблема: дает ли член (7.1) конечный, калибровочно-зависимый вклад в декремент нелинейного затухания Ландау? Ответ на этот вопрос будет нами дан во второй части нашей работы.

Рассмотрим теперь оставшиеся члены в (6.7). Вычислим свертку с ресуммированной 4-глюонной вершиной ${ }^{*} \Gamma_{4}$. Вычисления с использованием эффективных тождеств Уорда $(6.8),(6.9)$ и соотношений $(6.10),(7.2)$ приводят к выражению

$$
\begin{aligned}
& \left.{ }^{*} \Gamma^{\mu \sigma \lambda \nu}\left(k,-k_{2}, k_{1},-k_{3}\right) \bar{u}_{\mu}(k) \bar{u}_{\lambda}\left(k_{1}\right) \bar{u}_{\nu}\left(k_{3}\right) \bar{u}_{\sigma}\left(k_{2}\right)\right|_{\text {on-shell }}= \\
& \quad=k^{2} k_{1}^{2} k_{2}^{2} k_{3}^{2 *} \Gamma^{0000}\left(k,-k_{2}, k_{1},-k_{3}\right)+\Xi\left(k,-k_{2}, k_{1},-k_{3}\right),
\end{aligned}
$$


где

$$
\begin{aligned}
\Xi( & \left.,-k_{2}, k_{1},-k_{3}\right)=\left[\left(k_{1}^{2} k_{2}^{2}\left[\omega k_{3}^{2}+\omega_{3} k^{2}\right]^{*} \Gamma^{000}\left(k-k_{3}, k_{1},-k_{2}\right)-\right.\right. \\
& \left.\left.-\omega \omega_{3} k_{1}^{2} k_{2}^{2} k_{3 \nu}{ }^{*} \Gamma^{\nu 0}\left(k-k_{3}, k_{1},-k_{2}\right)\right)+\left(k \leftrightarrow k_{3}\right)+\left(k_{1} \leftrightarrow k\right)+\left(k_{1} \leftrightarrow k, k_{2} \leftrightarrow k_{3}\right)\right]+ \\
& +\left[\left(\left(\omega k_{3}^{2}+\omega_{3} k^{2}\right)\left(\omega_{2} k_{1}^{2}+\omega_{1} k_{2}^{2}\right) \mathcal{D}^{-100}\left(-k_{1}+k_{2}\right)-\right.\right. \\
& -\omega_{1} \omega_{2}\left(\omega k_{3}^{2}+\omega_{3} k^{2}\right) k_{1 \lambda} \mathcal{D}^{-10 \lambda}\left(-k_{1}+k_{2}\right)- \\
& -\omega \omega_{3}\left(\omega_{2} k_{1}^{2}+\omega_{1} k_{2}^{2}\right) k_{3 \nu} \mathcal{D}^{-10 \nu}\left(-k_{1}+k_{2}\right)+ \\
& \left.\left.+\omega \omega_{1} \omega_{2} \omega_{3} k_{1 \lambda} k_{3 \nu} \mathcal{D}^{-1 \nu \lambda}\left(-k_{1}+k_{2}\right)\right)+\left(k_{2} \leftrightarrow k_{3}\right)\right] .
\end{aligned}
$$

Далее вычисляем свертки в (6.7) с членами, содержащими ${ }^{*} \Gamma_{3}$. Здесь мы приходим к выражению

$$
\begin{aligned}
&\left\{\mathcal{D}_{\rho \alpha}(\right.\left.\left.-k_{1}+k_{2}\right)^{*} \Gamma^{\mu \nu \rho}\left(k,-k_{3}, k_{1}-k_{2}\right)^{*} \Gamma^{\alpha \lambda \sigma}\left(-k_{1}+k_{2}, k_{1},-k_{2}\right)+\left(k_{2} \leftrightarrow k_{3}\right)\right\} \times \\
& \quad \times\left.\bar{u}_{\mu}(k) \bar{u}_{\lambda}\left(k_{1}\right) \bar{u}_{\nu}\left(k_{3}\right) \bar{u}_{\sigma}\left(k_{2}\right)\right|_{\text {on-shell }}= \\
&=k^{2} k_{1}^{2} k_{2}^{2} k_{3}^{2}\left\{\mathcal{D}_{\rho \alpha}\left(-k_{1}+k_{2}\right)^{*} \Gamma^{00 \rho}\left(k,-k_{3}, k_{1}-k_{2}\right) \times\right. \\
&\left.\quad \times{ }^{*} \Gamma^{\alpha 00}\left(-k_{1}+k_{2}, k_{1},-k_{2}\right)+\left(k_{2} \leftrightarrow k_{3}\right)\right\}+\Xi\left(k,-k_{2}, k_{1},-k_{3}\right) .
\end{aligned}
$$

Отметим, что стояшая в правой части (7.4) функция $\Xi$ та же самая, что и в (7.3). Вычитая (7.4) из (7.3), приходим к искомому выражению, значительно более простому, чем исходное выражение (6.7),

$$
\begin{aligned}
& { }^{*} \widetilde{\Gamma}\left(k,-k_{2}, k_{1},-k_{3}\right)=k^{2} k_{1}^{2} k_{2}^{2} k_{3}^{2}\left\{{ }^{*} \Gamma^{0000}\left(k,-k_{2}, k_{1},-k_{3}\right)-\right. \\
& -\mathcal{D}_{\rho \alpha}\left(k-k_{3}\right)^{*} \Gamma^{00 \rho}\left(k,-k_{3}, k_{1}-k_{2}\right)^{*} \Gamma^{\alpha 00}\left(-k_{1}+k_{2}, k_{1},-k_{2}\right)- \\
& \left.-\mathcal{D}_{\rho \alpha}\left(k-k_{2}\right)^{*} \Gamma^{00 \rho}\left(k,-k_{2}, k_{1}-k_{3}\right)^{*} \Gamma^{\alpha 00}\left(-k_{1}+k_{3}, k_{1},-k_{3}\right)\right\} \text {. }
\end{aligned}
$$

Рассмотрим теперь структуру $* \widetilde{\Gamma}$ в $A_{0}$-калибровке. Для этого заменим в $(6.7)$ все векторы $\bar{u}_{\mu}$ на $\tilde{u}_{\mu}$, а пропагатор в ковариантной калибровке - на пропагатор в $A_{0}$-калибровке (6.6). Свертка с эффективной 4-глюонной вершиной приводит к выражению

$$
\begin{aligned}
& \left.{ }^{*} \Gamma^{\mu \sigma \lambda \nu}\left(k,-k_{2}, k_{1},-k_{3}\right) \tilde{u}_{\mu}(k) \tilde{u}_{\lambda}\left(k_{1}\right) \tilde{u}_{\nu}\left(k_{3}\right) \tilde{u}_{\sigma}\left(k_{2}\right)\right|_{\text {on-shell }}= \\
& \quad=k^{2} k_{1}^{2} k_{2}^{2} k_{3}^{2}\left\{{ }^{*} \Gamma^{0000}\left(k,-k_{2}, k_{1},-k_{3}\right)+\widetilde{\Xi}\left(k,-k_{2}, k_{1},-k_{3}\right)\right\},
\end{aligned}
$$

где

$$
\begin{aligned}
\widetilde{\Xi}(k, & \left.-k_{2}, k_{1},-k_{3}\right)=\left[\left(\omega_{1} \omega_{2}\left(\omega+\omega_{3}\right)^{*} \Gamma^{000}\left(k-k_{3}, k_{1},-k_{2}\right)-\right.\right. \\
& \left.\left.-\omega_{1} \omega_{2} k_{3 \nu}{ }^{*} \Gamma^{\nu 00}\left(k-k_{3}, k_{1},-k_{2}\right)\right)+\left(k \leftrightarrow k_{3}\right)+\left(k_{1} \leftrightarrow k\right)+\left(k_{1} \leftrightarrow k, k_{2} \leftrightarrow k_{3}\right)\right]+ \\
& +\left[\left(\left(\omega_{1}+\omega_{2}\right)\left(\omega+\omega_{3}\right) \mathcal{D}^{-100}\left(-k_{1}+k_{2}\right)-\left(\omega+\omega_{3}\right) k_{1 \lambda} \mathcal{D}^{-10 \lambda}\left(-k_{1}+k_{2}\right)-\right.\right. \\
& \left.\left.-\left(\omega_{1}+\omega_{2}\right) k_{3 \nu} \mathcal{D}^{-10 \nu}\left(-k_{1}+k_{2}\right)+k_{1 \lambda} k_{3 \nu} \mathcal{D}^{-1 \nu \lambda}\left(-k_{1}+k_{2}\right)\right)+\left(k_{2} \leftrightarrow k_{3}\right)\right] .
\end{aligned}
$$


Свертки с членами, содержащими ${ }^{*} \Gamma_{3}$, дают

$$
\begin{aligned}
\left\{\widetilde{\mathcal{D}}_{\rho \alpha}(\right. & \left.\left.-k_{1}+k_{2}\right)^{*} \Gamma^{\mu \nu \rho}\left(k,-k_{3}, k_{1}-k_{2}\right)^{*} \Gamma^{\alpha \lambda \sigma}\left(-k_{1}+k_{2}, k_{1},-k_{2}\right)+\left(k_{2} \leftrightarrow k_{3}\right)\right\} \times \\
\quad & \times\left.\tilde{u}_{\mu}(k) \tilde{u}_{\lambda}\left(k_{1}\right) \tilde{u}_{\nu}\left(k_{3}\right) \tilde{u}_{\sigma}\left(k_{2}\right)\right|_{\text {on-shell }}= \\
= & k^{2} k_{1}^{2} k_{2}^{2} k_{3}^{2}\left\{\widetilde{\mathcal{D}}_{\rho \alpha}\left(-k_{1}+k_{2}\right)^{*} \Gamma^{00 \rho}\left(k,-k_{3}, k_{1}-k_{2}\right)^{*} \Gamma^{\alpha 00}\left(-k_{1}+k_{2}, k_{1},-k_{2}\right)+\right. \\
\quad & \left.+\left(k_{2} \leftrightarrow k_{3}\right)+\widetilde{\Xi}\left(k,-k_{2}, k_{1},-k_{3}\right)\right\} .
\end{aligned}
$$

Вычитая (7.7) из (7.6), приходим к аналогичному выражению (7.5). Таким образом, нами показано, что, по крайней мере, в классе ковариантной и $A_{0}$-калибровок декремент нелинейного затухания Ландау (6.1) (точнее его часть, не зависяшая от калибровочного параметра) калибровочно-инвариантен.

\section{8. ЗАКЛЮЧЕНИЕ}

Рассмотрим более подробно приближения, которые мы используем в данной статье. Фактически здесь применяются два различных уровня аппроксимаций. Первый из них связан с использованием обычного подхода, развитого в абелевой плазме применительно к КГП, т.е. со стандартным разложением тока по амплитуде колебаний и вычислением взаимодействуюшего поля в виде ряда теории возмушений по свободному полю $A^{(0)}$ (точнее, по $g A^{(0)}$ ). Однако, в отличие от абелевой плазмы, в нашем случае уже из учета первых двух нелинейных порядков цветного тока возникает существенно больше членов, определяюших нелинейное рассеяние волн. Здесь мы использовали второй уровень аппроксимации, связанный с представлениями, идущими из работ по жестким температурным петлям, а точнее, систему оценок порядков различных членов, развитую в работе [18]. Эта система оценок позволяет среди множества полученных членов выделить главные по константе взаимодействия. Все они оказались чисто неабелевыми в полном соответствии с выводами [18].

Однако использование результатов работы [18] в нашем случае является не совсем последовательным. Так, например, в операторе ковариантного дифференцирования, в отличие от [18], мы считаем, что $\partial / \partial X^{\mu}$ и $g A_{\mu}(X)$ имеют разные порядки. В тензоре напряженности калибровочного поля мы различаем линейную и нелинейную части и т.п. Поэтому вычислительная схема, используемая в данной работе, страдает тем недостатком, что она явно нарушает неабелеву калибровочную симметрию теории на каждом конечном шаге приближенных вычислений.

Это действительно так, если считать, как и в [18], что амплитуда мягких колебаний $A_{\mu}^{a}(X)$ имеет порядок $T$ или в представлении Фурье $A_{\mu}^{a}(k) \sim 1 / g(g T)^{3}$. В этом случае, используя найденные явно выражения для членов в разложении цветного тока (3.2), $(4.4),(4.9)$ и оценки $(4.7),(4.12)$, имеем

$$
j_{\mu}^{\mathrm{T}(1)}(k) \sim j_{\mu}^{\mathrm{T}(2)}(k) \sim j_{\mu}^{\mathrm{T}(3)}(k) \sim \cdots \sim \frac{1}{g^{2} T},
$$


т.е. каждый член в разложении (2.14) дает одинаковый вклад и поэтому необходимо суммировать весь ряд. Тем самым восстанавливается калибровочная симметрия, и мы приходим к известному выражению $[18,19]$ для цветного тока (см. также [37]), которое в координатном представлении имеет вид

$$
j^{a \mu}(X)=3 \omega_{\mathrm{pl}}^{2} \int \frac{d \Omega}{4 \pi} v^{\mu} W^{a}(X ; v)
$$

где

$$
W^{a}(X ; v)=\int_{0}^{\infty} d u U^{a b}(X, X-v u) F^{b 0 \mu}(X-v u) v_{\mu}
$$

и $U(X, Y)$ - оператор параллельного смешения.

В данной работе мы ограничились учетом только конечного числа членов ряда в разложении (2.14). Это налагает более жесткие ограничения на величину амплитуды колебаний: $A_{\mu}^{a}(X) \sim g T\left(A_{\mu}^{a}(k) \sim 1 /(g T)^{3}\right)$. В этом случае имеем оценки

$$
j_{\mu}^{\mathrm{T}(1)}(k) \sim \frac{1}{g T}, \quad j_{\mu}^{\mathrm{T}(2)}(k) \sim \frac{1}{T}, \quad j_{\mu}^{\mathrm{T}(3)}(k) \sim \frac{g}{T}, \quad \ldots,
$$

каждый следуюший член в разложении тока подавляется по константе взаимодействия по сравнению с предыдущим, и использование теории возмущений становится оправданным. Восстановление калибровочной симметрии происходит за счет учета слабой корреляции при вычислении среднего от трех случайных полей (раздел 5) в слабонеоднородной и слабонестационарной КГП. При этом мы получаем выражение для декремента нелинейного затухания Ландау (6.1), весьма близкое по внешнему виду соответствуюшему глюонному декременту затухания в HTL-аппроксимации [4].

Отметим также, что в работе [18] изучается поведение среднего поля $\left\langle A_{\mu}^{a}(X)\right\rangle$, которое в нашем случае равно нулю в силу предполагаемого стохастического характера фазы колебаний. Поэтому мы изучаем поведение коррелятора от двух случайных полей и в конечном счете поведение функции распределения числа плазмонов $N^{l}(t, \mathbf{x}, \mathbf{k})$. Импульс $\mathbf{k}$ имеет порядок $g T$, в то время как медленные переменные $t$ и $\mathbf{x}$ изменяются на шкале порядка $1 /\left(g^{2} T\right)$. Последнее обстоятельство проше всего увидеть из кинетического уравнения (5.16). Как будет показано во второй части нашей работы, декремент нелинейного затухания Ландау имеет порядок $g^{2} T$, поэтому, для того чтобы левая и правая части кинетического уравнения (5.16) были одного порядка, необходимо считать $\partial_{t}, \partial_{\mathbf{x}} \sim g^{2} T$. Таким образом, кинетическое уравнение (5.16) и исходные квазиклассические динамические уравнения (2.4) описывают поведение системы при различных хорошо разделенных при $g \ll 1$ масштабах как по импульсу, так и по координате.

Благодарности. Работа выполнена при поддержке Российского фонда фундаментальных исследований (проект № 97-02-16065). 


\section{Список литературы}

[1] E. Braaten, R. D. Pisarski. Nucl. Phys. B. 1990. V. 337. P. 569.

[2] J. Frenkel, J. C. Taylor. Nucl. Phys. B. 1990. V. 334. P. 199.

[3] R. D. Pisarski. Phys. Rev. Lett. 1989. V. 63. P. 1129.

[4] E. Braaten, R. D. Pisarski. Phys. Rev. D. 1990. V. 42. P. 2156.

[5] R. Kobes, G. Kunstatter, K. Mak. Phys. Rev. D. 1992. V. 45. P. 4632.

[6] E. Braaten, R. D. Pisarski. Phys. Rev. D. 1992. V. 46. P. 1829.

[7] V. V. Lebedev, A. V. Smilga. Ann. Phys. (N. Y.). 1990. V. 202. P. 229.

[8] V. V. Lebedev, A. V. Smilga. Phisica A. 1992. V. 181. P. 187.

[9] U. Heinz. Phys. Rev. Lett. 1983. V. 51. P. 351.

[10] U. Heinz. Ann. Phys. (N. Y. ). 1985. V. 161. P. 48; 1986. V. 168. P. 148.

[11] В. П. Силин, В. Н. Урсов. ДАН СССР. 1985. Т. 283. С. 594.

[12] H.-Th. Elze, M. Gyulassy, D. Vasak. Phys. Lett. B. 1986. V. 177. P. 402.

[13] H.-Th. Elze, M. Gyulassy, D. Vasak. Nucl. Phys. B. 1986. V. 276. P. 706.

[14] Ю. А. Марков, М. А. Маркова. ТМФ. 1997. Т. 111. С. 263.

[15] В. П. Силин. ЖКЭТФ. 1960. Т. 38. С. 1577.

[16] P.F. Kelly, Q. Liu, C. Lucchesi, C. Manuel. Phys. Rev. D. 1994. V. 50. P. 4209.

[17] H. Heiselberg, C. J. Pethick. Phys. Rev. D. 1993. V. 47. P. R769.

[18] J. P. Blaizot, E. Iancu. Nucl. Phys. B. 1994. V. 417. P. 608.

[19] J. P. Blaizot, E. Iancu. Nucl. Phys. B. 1994. V. 421. P. 565.

[20] Z. Xiaofei, L. Jiarong. Phys. Rev. C. 1995. V. 52. P. 964.

[21] U. Heinz, P. J. Siemens. Phys. Lett. B. 1985. V. 158. P. 11.

[22] А. Г. Ситенко. Флуктуации и нелинейное взаимодействие волн в плазме. Киев: Наукова Думка, 1977.

[23] Б. Б. Кадомцев. Турбулентность плазмы. В сб.: Вопросы теории плазмы. Вып. 4. Ред. М. А. Леонтович. М.: Атомиздат, 1964. С. 188.

[24] Ю. Л. Климонтович. Статистическая теория неравновесных процессов в плазме. М.: Изд-во МГУ, 1964.

[25] В. Н. Цытович. Нелинейные эффекты в плазме. М.: Наука, 1967.

[26] В. Н. Цытович. Теория турбулентной плазмы. М.: Атомиздат, 1971.

[27] В. В. Пустовалов, В. П. Силин. Тр. ФИАН. 1972. Т. 61. С. 38.

[28] О.К. Калашников, В. В. Климов. ЯФ. 1980. Т. 31. С. 1357.

[29] В. В. Климов. ЖЭЭТФ. 1982. Т. 82. С. 336.

[30] H. A. Weldon. Phys. Rev. D. 1982. V. 26. P. 1394.

[31] K. Kajantie, J. Kapusta. Ann. Phys. (N. Y. ). 1985. V. 160. P. 477.

[32] U. Heinz, K. Kajantie, T. Toimela. Ann. Phys. (N. Y. ). 1987. V. 176. P. 218.

[33] А. В. Смилга. ЯФ. 1994. Т. 57. С. 550.

[34] R. Baier, G. Kunstatter, D. Schiff. Nucl. Phys. B. 1992. V. 388. P. 287.

[35] R. Baier, G. Kunstatter, D. Schiff. Phys. Rev. D. 1992. V. 45. P. R4381.

[36] H. Nakkagawa, A. Niegawa, B. Pire. Phys. Lett. B. 1992. V. 294 . P. 396.

[37] R. Jackiw, V.P. Nair. Phys. Rev. D. 1993. V. 48. P. 4991.

Поступила в редакцию 12. VIII.1999 г., после доработки 5.XI.1999 г. 